\title{
The Ground State Energy of Dilute Bose Gas in Potentials with Positive Scattering Length
}

\author{
Jun Yin \\ Department of Mathematics, Harvard University, \\ Email: JYin@math.harvard.edu
}

August 29th, 2008

\begin{abstract}
The leading term of the ground state energy/particle of a dilute gas of bosons with mass $m$ in the thermodynamic limit is $2 \pi \hbar^{2} a \varrho / m$ when the density of the gas is $\varrho$, the interaction potential is non-negative and the scattering length $a$ is positive. In this paper, we generalize the upper bound part of this result to any interaction potential with positive scattering length, i.e, $a>0$ and the lower bound part to some interaction potentials with shallow and/or narrow negative parts.
\end{abstract}

\section{Introduction and main theorems}

In Dyson's work [9] and Lieb, Yngvason and Seiringer's work [7, 6], it is rigorously proved that the leading term of the ground state energy/particle of a three dimensional dilute bose gas of mass $m$ in the thermodynamic limit with density $\varrho$ is $2 \pi \hbar^{2} a \varrho / m$, i.e.,

$$
e(\varrho, m)=2 \pi \hbar^{2} a \varrho / m(1+o(1)) \text { if } a^{3} \varrho \ll 1
$$

where they assumed that the interaction potential is non-negative, the scattering length $a$ is positive. This result is generalized to a two dimensional dilute bose gas in [8]. In this paper, first, in Theorem 1, we generalize the upper bound part of (1.1) to general interaction potentials $v$ with positive scattering length. On the other hand, for the lower bound on the ground energy, it was conjectured in [7] that the lower bound part of (1.1) should hold if the scattering length is positive and $v$ has no $N$ body bound states for any $N$. Recently, it is proved in [11] that in some cases with partly shallow negative potential the lower bound part of (1.1) holds. In Theorem 2, we introduce a different method for the lower bound on (1.1) when $v$ can have shallow and/or narrow negative components and provide better(smaller) error term.

$* * *$

(C) 2008 by the author. This paper may be reproduced, in its entirety, for non-commercial purposes. 
We begin with describing the questions more precisely. We write the Hamiltonian of a system of $N$ interacting bosons which are restricted to a cubic box of volume $\Lambda=L^{3}$ in the following way (in units where $\hbar=2 m=1$ ):

$$
H_{N} \equiv \sum_{i=1}^{N}-\Delta_{i}+\sum_{1 \leq i<j \leq N} v^{a}\left(x_{i}-x_{j}\right)
$$

Here $\Delta$ denotes the Laplacian on $\Lambda$ with periodic boundary condition and $v^{a}$ is a scaled interaction potential, i.e.,

$$
v^{a}(x)=a^{-2} \cdot v(x / a), \quad a>0
$$

The pair interaction potential $v$ is spherically symmetric and supported on the set $\left\{x \in \mathbb{R}^{3}:|x| \leq R_{0}\right\}$ for some $R_{0}>0$.

DEFINITION 1 (Scattering Length). Assume that $w$ is a pair spherically symmetric interaction potential with compact support. Denote $E[\phi]$ as the energy of the complex-valued function $\phi$ on $\mathbb{R}^{3}$ as follows,

$$
E[\phi]=\int_{\mathbb{R}^{3}}|\nabla \phi(x)|^{2}+\frac{1}{2} w(x)|\phi(x)|^{2} d x .
$$

Define the scattering length $S L(w)$ of potential $w$ as the following minimum energy.

$$
S L(w) \equiv \min _{\phi}\left\{\frac{1}{4 \pi} E[\phi]: \lim _{|x| \rightarrow \infty} \phi(x)=1\right\}
$$

Note: If $S L(w)>-\infty$, one can easily prove that the Hamiltonian $-\Delta+\frac{1}{2} w$ has no bound state. In particular, when $w \geq 0$, we have $S L(w) \geq 0$ and $w$ has no bound state. One can see that this definition is equivalent to the definition of scattering length in [5] when $w>0$.

With the relation between $v$ and $v^{a}$ in (1.3), we can assume that

$$
S L(v)=1, \quad S L\left(v^{a}\right)=a
$$

Let $f_{1}(x)$ be the solution of the zero-energy scattering equation of $v$, i.e.,

$$
-\Delta f_{1}(x)+\frac{1}{2} v(x) f_{1}(x)=0
$$

then we have that $f_{a}(x) \equiv f_{1}(x / a)$ is the solution of the following zero-energy scattering equation of $v^{a}$.

$$
-\Delta f_{a}(x)+\frac{1}{2} v^{a}(x) f_{a}(x)=0
$$

As in [5], one can prove that if $f_{a}$ is normalized as $\lim _{|x| \rightarrow \infty} f_{a}(x)=1$, then

$$
f_{a}(x)=1-a / x, \text { for }|x|>R_{0} a
$$

In this paper, we are interested in the ground energy $E(N, \Lambda)$ of $H_{N}$ in the thermodynamic limit that $\Lambda \rightarrow \infty, N \rightarrow \infty$ and $N / \Lambda=\varrho$. Low density means that the average inter-particle distance $\varrho^{-1 / 3}$ is much larger than the scattering length $a$, i.e. $a^{3} \varrho \ll 1$.

First, we state that for any fixed $v$, the upper bound on (1.1) holds for the dilute bose gas. 
THEOREM 1. Fix $v$ with $S L[v]=1$ and $v^{a}$ satisfying (1.3). Let $f_{1}$ be the solution of zero-energy equation of $v$ and normalized as: $f_{1}(\infty)=1$. In the thermodynamic limit, $\lim _{N \rightarrow \infty} N / \Lambda=\varrho$, we have the following upper bound on $E(N, \Lambda)$, which is the ground energy of $H_{N}$ in (1.2),

$$
\limsup _{N \rightarrow \infty} \frac{E(N, \Lambda)}{4 \pi a \varrho N} \leq 1+\text { const. }\left(a^{3} \varrho\right)^{1 / 4}
$$

for some constant depending on $\left\|f_{1}\right\|_{\infty}$, provided that $\frac{4 \pi}{3} a^{3} \varrho \leq 1$.

Note: So far, the best proof of the error term on upper bound, when $v \geq 0$, is $O\left(a^{3} \varrho\right)^{1 / 3}$, as in [5].

On the other hand, for the lower bound in (1.1), we prove that as long as $v$ has a positive core and is bounded from below, (1.1) holds when the negative part is small enough (shallow and/or narrow). In the appendix, we show that if $v^{a}$ is a continuous function on $\mathbb{R}^{3}$ and $H_{N}$ has no bound state for any $N, v^{a}$ satisfies the above two requirements, i.e.,

$$
v^{a}(0)>0, \quad \min v^{a}(r)>-\infty
$$

The above two inequalities (1.11) also hold when $v^{a}$ is stable [1] (the stability of potential is assumed in [11]).

THEOREM 2. We assume that $v(x)=v_{+}(x)+v_{-}(x), v_{+}(x) \geq 0, v_{-}(x) \geq-\lambda_{-}$, $\lambda_{-}>0$ and $v_{+}$has a positive core, i.e. $\exists r_{1}$, such that $v_{+}(x) \geq \lambda_{+}>0$ for $|x| \leq r_{1}$. Here $v_{-}$need not be negative.

There exist $c_{1}\left(R_{0} / r_{1}\right)$ and $c_{2}\left(R_{0} / r_{1}\right)$, which are greater than one and only depend on $R_{0} / r_{1}$, such that the following holds.

If there exists some positive number $t$ satisfying

$$
S L\left[c_{1}\left(R_{0} / r_{1}\right) \cdot\left(v+t v_{-}\right)\right] \geq 0 \text { and } \lambda_{+} \geq\left(1+t^{-1}\right) c_{2}\left(R_{0} / r_{1}\right) \cdot \lambda_{-},
$$

we have the following lower bound on $E(N, \Lambda)$,

$$
\liminf _{N \rightarrow \infty} \frac{E(N, \Lambda)}{4 \pi a \varrho N} \geq 1-\text { const. }\left(a^{3} \varrho\right)^{1 / 17},
$$

for some constant depending on $v_{+}$and $v_{-}$, provided that $\frac{4 \pi}{3} a^{3} \varrho$ is smaller than some constant depending on $v_{+}, v_{-}$and $t$.

Note: So far, the best estimation of the error term of the lower bound, when $v>0$, is also $O\left(a^{3} \varrho\right)^{1 / 17}$, as in [5].

This theorem implies the following two corollaries.

COROLLARY 1. Assume that

$$
v(x)=v_{+}(x)+\lambda_{-} v_{-}(x), v_{+}(x) \geq 0, \quad v_{-}(x) \geq-1
$$


and $v_{+}$has a positive core, i.e. $\exists r_{1}$ such that $v_{+}(x) \geq \lambda_{+}$for $|x| \leq r_{1}$. There exists $\lambda_{0}\left(r_{1}, R_{0}, \lambda_{+}, v_{-}\right)$such that, if $0 \leq \lambda_{-} \leq \lambda_{0}$, i.e., the potential is shallow enough, we have the following lower bound on $E(N, \Lambda)$,

$$
\liminf _{N \rightarrow \infty} \frac{E(N, \Lambda)}{4 \pi a \varrho N} \geq 1-\text { const. }\left(a^{3} \varrho\right)^{1 / 17}
$$

provided that $\frac{4 \pi}{3} a^{3} \varrho$ is smaller than some constant depending on $v_{+}$and $v_{-}$.

Proof. For fixed $R_{0}, r_{1}$ and $\lambda_{+}$, when $\lambda_{-}$is small enough, we have that

$$
S L\left[c_{1}\left(R_{0} / r_{1}\right)\left(v_{+}+2 \lambda_{-} v_{-}\right)\right] \geq 0 \text { and } \lambda_{+} \geq 2 c_{2}\left(R_{0} / r_{1}\right) \lambda_{-} .
$$

Using Theorem 2, with the choice $t=1$, we arrive at the desired result.

COROLLARY 2. Assume that

$$
v(x)=v_{+}(x)+v_{-}(x), \quad v_{+}(x) \geq 0 \geq v_{-}(x) \geq-\lambda_{-}
$$

and $v_{+}$has a positive core, i.e. $\exists r_{1}$ such that $v_{+}(x) \geq \lambda_{+}$for $|x| \leq r_{1}$. There exist $\lambda_{0}\left(R_{0} / r_{1}, \lambda_{-}\right)$and $\varepsilon\left(R_{0}, r_{1}, \lambda_{-}\right)$such that, if

$$
\lambda_{+} \geq \lambda_{0} \text { and } \int_{x \in \mathbb{R}^{3}}\left|v_{-}(x)\right| d x \leq \varepsilon\left(R_{0}, r_{1}, \lambda_{-}\right),
$$

we have the following lower bound on $E(N, \Lambda)$,

$$
\liminf _{N \rightarrow \infty} \frac{E(N, \Lambda)}{4 \pi a \varrho N} \geq 1-\text { const. }\left(a^{3} \varrho\right)^{1 / 17}
$$

provided that $\frac{4 \pi}{3} a^{3} \varrho$ is smaller than some constant depending on $v_{+}$and $v_{-}$.

Proof. We choose $\lambda_{0}=\max \left\{3,2 c_{2}\left(R_{0} / r_{1}\right)\right\} \lambda_{-}$, then we have that $\lambda_{+} \geq \lambda_{0} \geq 3 \lambda_{-}$, which implies that

$$
\left[v+v_{-}\right](x) \geq \lambda_{+} / 3 \geq 0 \text { for }|x| \leq r_{1}
$$

Then we claim that for any $n \geq 1$ and $\lambda_{+} \geq 3 \lambda_{-}$, there exists $\xi(n)>0$,

$$
\int_{\mathbb{R}^{3}}\left|v_{-}(x)\right| d x \leq \xi(n) \Rightarrow S L\left[n\left(v+v_{-}\right)\right] \geq 0 .
$$

To prove (1.21), we shall prove that there exists $\xi(n)>0$, if $\int_{\mathbb{R}^{3}}\left|v_{-}(x)\right| d x \leq \xi(n)$, for any non-negative radial function $f$,

$$
\int_{|x| \leq R_{0}}|\nabla f|^{2}(x)+\frac{n}{2}\left(v+v_{-}\right) f^{2}(x) d x \geq 0 .
$$

We can see, with (1.20),

$$
\begin{aligned}
& \int_{|x| \leq R_{0}}\left[|\nabla f|^{2}(x)+\frac{n}{2}\left(v+v_{-}\right) f^{2}(x)\right] d x \\
\geq & \int_{r_{1} \leq|x| \leq R_{0}}\left[|\nabla f|^{2}(x)-\left\|n v_{-}\right\|_{\infty} f^{2}(x)\right] d x \\
\geq & \int_{r_{1} \leq|x| \leq R_{0}}|\nabla f|^{2}(x) d x-n \lambda_{-} \int_{r_{1} \leq|x| \leq R_{0}}|f|^{2}(x) d x
\end{aligned}
$$


Hence, if (1.22) does not hold, the right side of (1.23) is less than 0. With Sobolev inequality and Schwarz's Inequality, we obtain that there exists $\eta(n)$ such that

$$
\left(\int_{r_{1} \leq|x| \leq R_{0}}|f(x)|^{4} d x\right)^{1 / 2} \leq \eta(n) \int_{r_{1} \leq|x| \leq R_{0}}|f|^{2}(x) d x
$$

On the other hand, with (1.20), $v+v_{-} \geq 2 v_{-}$and Schwarz's Inequality, we have that

$$
\begin{aligned}
& \int_{|x| \leq R_{0}}|\nabla f|^{2}(x)+\frac{n}{2}\left(v+v_{-}\right) f^{2}(x) d x \\
\geq & \int_{|x| \leq R_{0}}|\nabla f|^{2}(x)+\int_{|x| \leq r_{1}} \frac{n \lambda_{+}}{6} f^{2}(x)-\left.\left|\int_{r_{1} \leq|x| \leq R_{0}} n v_{-}(x)\right| f(x)\right|^{2} d x \mid \\
\geq & \int_{|x| \leq R_{0}}|\nabla f|^{2}(x)+\int_{|x| \leq r_{1}} \frac{n \lambda_{+}}{6} f^{2}(x) \\
& -n \eta(n)\left|\int_{r_{1} \leq|x| \leq R_{0}} v_{-}^{2}(x) d x\right|^{1 / 2} \int_{r_{1} \leq|x| \leq R_{0}}|f|^{2}(x) d x \\
\geq & \int_{|x| \leq R_{0}}|\nabla f|^{2}(x)+\int_{|x| \leq r_{1}} \frac{n \lambda_{+}}{6} f^{2}(x)-n \eta(n) \lambda_{-}\left\|v_{-}\right\|_{1}^{1 / 2} \int_{|x| \leq R_{0}}|f|^{2}(x) d x
\end{aligned}
$$

Thus, for $n \geq 1$, if

$$
\left\|v_{-}\right\|_{1}^{1 / 2} \leq(\xi(n))^{1 / 2} \equiv \frac{1}{n \eta(n)} \cdot \min _{f} \frac{\int_{|x| \leq R_{0}}|\nabla f|^{2}(x)+\int_{|x| \leq r_{1}} \frac{n \lambda_{+}}{6} f^{2}(x) d x}{\int_{|r| \leq R_{0}}|f|^{2}(x) d x},
$$

the inequality (1.22) holds. We note that it is easy to see that $\xi(n)>0$. Hence we arrive at the desired result (1.21). At last, choosing

$$
\varepsilon\left(R_{0}, r_{1}, \lambda_{-}\right)=\xi\left(c_{1}\left(R_{0} / r_{1}\right)\right)
$$

and using the result of Theorem 2 with $t=1$, we arrive at the desired result (1.19).

Remark: Compared with the result of [11, we improve the error term (It was $\left(a^{3} \varrho\right)^{1 / 31}$ in [1] $)$ and generalize the shapes of potentials, i.e., the negative part of potential can be shallow and/or narrow. In particular, there is no restriction on the depth of the interaction potential $v$, i.e. for $\forall \lambda_{-}>0$, there $\exists v$ satisfying $\min _{x \in \mathbb{R}^{3}} v(x)<-\lambda_{-}$and Theorem 2 holds.

\section{Proofs}

\subsection{Proof of Theorem one}

Proof. As usual, to prove the upper bound on the ground state energy, we only need to construct a sequence of trial states $\Psi_{N, \Lambda}$ satisfying

$$
\limsup _{N \rightarrow \infty} \frac{\left\langle\Psi\left|H_{N}\right| \Psi\right\rangle}{N\langle\Psi \mid \Psi\rangle} \leq 4 \pi a \varrho(1+\text { const. } Y)
$$


for some constant that depends only on $\left\|f_{1}\right\|_{\infty}$. Here we denote $Y$ as

$$
Y \equiv\left(\frac{4 \pi}{3} a^{3} \varrho\right)^{1 / 4}
$$

Following the ideas in [9, 6], we construct the trial state of the following form,

$$
\Psi_{N}=\prod_{p=1}^{N} F_{p}
$$

In 9 ] $F_{p}$ depends on the the nearest particle to the $x_{p}$ among all the $x_{i}$ with $i<p$, i.e.,

$$
F_{p}=f\left(t_{p}\right), \quad t_{p}=\min _{i<p}\left\{\left|x_{i}-x_{p}\right|\right\}
$$

via the function $f$ which is very close to the zero energy scattering solution and satisfies

$$
0 \leq f \leq 1, \quad f^{\prime} \geq 0
$$

Hence in [9], $F_{p}$ has the following property

$$
F_{p, i} \cdot f\left(\left|x_{p}-x_{i}\right|\right) \leq F_{p} \leq F_{p, i}
$$

Here $F_{p, i}$ is defined in [9] as the value that $F_{p}$ would take if the point $x_{i}$ were omitted from consideration.

But in our case where the potential has a negative part, the zero energy scattering solution $f_{a}$ of $v^{a}$ may not be an increasing function or bounded by 1 (if it was, the proof would be much simpler). Hence we do not have the property (2.6). For this reason, our choice of $F_{p}$ will be more complicated. Our $F_{p}$ depends on all particles near the $x_{p}$, not just the nearest.

We remark that the function $F_{p}$ should have following properties.

1. $F_{p}$ is a continuous function of $x_{i}(1 \leq i \leq N)$.

2. When $\left|x_{i}-x_{p}\right|$ is large enough, the position of $x_{i}$ does not effect $F_{p}$, i.e., $\nabla_{x_{i}} F_{p}=0$.

3. $F_{p}$ has a similar property as (2.6).

First we define $\theta_{r}(x)$ as the characteristic function of the set $\{x:|x| \leq r\}$ and $\bar{\theta}_{r} \equiv 1-\theta_{r}$. Choosing $b=a / Y$, we have

$$
a / b=\frac{4 \pi}{3} b^{3} N / \Lambda=Y .
$$

Without loss of generality, we assume that $b>\max \left\{2 R_{0} a, 4 a\right\}$, as in [9, 5]. We define $f(x)$ as

$$
f(x)= \begin{cases}f_{a}(x) / f_{a}(b) & b \geq|x| \geq 0 \\ 1 & \text { otherwise }\end{cases}
$$


Here $f_{a}$ is the zero energy scattering solution of $v^{a}$, as in (1.8). With the equation (1.9), we note that

$$
f(x)=\frac{1-a /|x|}{1-a / b}, \text { for } b \geq|x| \geq R_{0} a .
$$

Let $\widetilde{R}=\max \left\{R_{0} a, 2 a\right\}$, which implies that $f(\widetilde{R})>\frac{1}{2}$. We define $\Theta_{p}^{\text {in }}$, $\Theta_{p}^{\text {out }}(1<p \leq$ $N)$ as

$$
\Theta_{p}^{i n} \equiv \prod_{j<p} \theta_{\widetilde{R}}\left(x_{j}-x_{p}\right), \quad \Theta_{p}^{\text {out }} \equiv \prod_{j<p} \bar{\theta}_{\widetilde{R}}\left(x_{j}-x_{p}\right)
$$

We can see that $\Theta_{p}^{i n}=1$ when $\left|x_{j}-x_{p}\right| \leq \widetilde{R}$ for all $j<p$ and $\Theta_{p}^{\text {out }}=1$ when $\left|x_{j}-x_{p}\right|>\widetilde{R}$ for all $j<p$. With $\Theta_{p}^{\text {in }}$ and $\Theta_{p}^{\text {out }}$, we can define $r_{p}\left(x_{1}, \cdots, x_{N}\right)$ and $R_{p}\left(x_{1}, \cdots, x_{N}\right)$ as follows, $\left(x_{i} \in[0, L]^{3}, i=1, \cdots N\right)$

$$
\begin{aligned}
& r_{p} \equiv \\
& \left(1-\Theta_{p}^{\text {out }}\right) \cdot \min _{i<p}\left\{\left|x_{i}-x_{p}\right|: f\left(x_{i}-x_{p}\right)=\min _{j<p}\left\{f\left(x_{j}-x_{p}\right):\left|x_{j}-x_{p}\right| \leq \widetilde{R}\right\}\right\} \\
& R_{p} \equiv \widetilde{R} \cdot \Theta_{p}^{i n}+\left(1-\Theta_{p}^{i n}\right) \times \min _{i<p}\left\{\left|x_{i}-x_{p}\right|:\left|x_{i}-x_{p}\right|>\widetilde{R}\right\}
\end{aligned}
$$

With the definition of $R_{p}$ and (2.9), we have that

1. $f\left(R_{p}\right) \leq f\left(x_{j}-x_{p}\right)$ for any $j<p$ satisfying $\left|x_{j}-x_{p}\right|>\widetilde{R}$,

2. $R_{p} \leq\left|x_{j}-x_{p}\right|$ for any $j<p$ satisfying $\left|x_{j}-x_{p}\right|>\widetilde{R}$.

3. $R_{p} \geq \widetilde{R}$

4. When $\Theta_{p}^{i n}=0$, there exists $j_{p}$ such that $\left|x_{j_{p}}-x_{p}\right|=R_{p}$

Similarly, we have

1. $f\left(r_{p}\right) \leq f\left(x_{j}-x_{p}\right)$ for any $j<p$ satisfying $\left|x_{j}-x_{p}\right| \leq \widetilde{R}$,

2. $r_{p} \leq\left|x_{j}-x_{p}\right|$ for any $j<p$ satisfying $\left|x_{j}-x_{p}\right| \leq \widetilde{R}$ and $f\left(x_{j}-x_{p}\right)=f\left(r_{p}\right)$.

3. $r_{p} \leq \widetilde{R}$

4. When $\Theta_{p}^{\text {out }}=0$, there exists $i_{p}$ such that $\left|x_{i_{p}}-x_{p}\right|=r_{p}$

Then, we define a continuous function $T$ on $\mathbb{R}$ as follows

$$
T(|x|)= \begin{cases}1 & 2 \widetilde{R} \geq|x| \\ \left(|x|^{-1}-b^{-1}\right)\left(2 \widetilde{R}^{-1}-b^{-1}\right)^{-1} & b \geq|x| \geq 2 \widetilde{R} \\ 0 & |x| \geq b\end{cases}
$$


At last we define $F_{p}\left(x_{1}, \cdots, x_{N}\right)$ on $[0, L]^{3 N}$ as follows $(1<p \leq N)$,

$$
F_{p} \equiv \begin{cases}f\left(r_{p}\right) & \Theta_{p}^{\text {in }}=1 \\ f\left(R_{p}\right) & \Theta_{p}^{\text {out }}=1 \\ f\left(r_{p}\right)+T\left(R_{p}\right)\left[f\left(R_{p}\right)-f\left(r_{p}\right)\right]_{-} & \text {otherwise }\end{cases}
$$

and $F_{1}=1$. Here $[\cdot]_{-}$denotes the negative part, i.e., $[x]_{-}=x$ when $x<0$ and $[x]_{-}=0$ when $x \geq 0$. We note that for any $x$

$$
[x]_{-} \leq 0
$$

Note: If $v \geq 0$, it is well known that $f$ is an increasing function, which implies the $F_{p}$ we defined is equal to the $F_{p}$ in $[9]$.

One can prove that $F_{p}$ is a continuous function of $\left(x_{1}, \cdots, x_{N}\right)$ by checking that, for any $j \neq p>1$ and fixed $x_{1}, \cdots, x_{j-1}, x_{j+1}, \cdots, x_{N}, F_{p}$ is a continuous function of $x_{j}$. First we can see that it is trivial for $j>p$, since $F_{p}$ is independent of $x_{j}$ when $j>p$. For $j<p$, it only remains to check that $F_{p}$ is continuous when $x_{j}$ moves from $\left|x_{j}-x_{p}\right|=\widetilde{R}$ to $\left|x_{j}-x_{p}\right|=\widetilde{R}+0^{+}$. One can see that when $\left|x_{j}-x_{p}\right|=\widetilde{R}$, $f\left(R_{p}\right) \geq f(\widetilde{R})=f\left(x_{j}-x_{p}\right) \geq f\left(r_{p}\right)$, so $F_{p}=f\left(r_{p}\right)$, i.e.

$$
F_{p}=\min \left\{\min _{k: k \neq j, k<p}\left\{f\left(x_{k}-x_{p}\right):\left|x_{k}-x_{p}\right| \leq \widetilde{R}\right\}, f\left(x_{j}-x_{p}\right)\right\}
$$

On the other hand, when $\left|x_{j}-x_{p}\right|=\widetilde{R}+0^{+} \leq 2 \widetilde{R}$, we can see that $R_{p}=\left|x_{j}-x_{p}\right|$, $T\left(R_{p}\right)=1$ and $f\left(R_{p}\right)=f(\widetilde{R})+0^{+}$. Hence,

$$
F_{p}=\min \left\{\min _{k: k \neq j, k<p}\left\{f\left(x_{k}-x_{p}\right):\left|x_{k}-x_{p}\right| \leq \widetilde{R}\right\}, f\left(x_{j}-x_{p}\right)\right\}
$$

Hence we arrive at the desired result that $F_{p}$ is continuous function.

We can also see that $F_{p}$ is non-negative and bounded as follows

$$
M \equiv\left\|F_{p}\right\|_{\infty}=\|f\|_{\infty} \leq(1-a / b)^{-1}\left\|f_{a}\right\|_{\infty}=(1-a / b)^{-1}\left\|f_{1}\right\|_{\infty} \leq 2\left\|f_{1}\right\|_{\infty} .
$$

Here we use the fact $f_{a}(x)=f_{1}(x / a)$.

By the definition of $F_{p}$, one can see that $F_{p}=1$ when $\prod_{q<p} \bar{\theta}_{b}\left(x_{p}-x_{q}\right)=1$ and $F_{p} \leq 1$ when $\prod_{q<p} \bar{\theta}_{\widetilde{R}}\left(x_{p}-x_{q}\right)=1$, so

$$
1-\sum_{q<p} \theta_{b}\left(x_{p}-x_{q}\right) \leq F_{p} \leq 1+\sum_{q<p}(M-1) \theta_{\widetilde{R}}\left(x_{p}-x_{q}\right)
$$

We now construct the state functions $\Phi_{k}$ as follows $(1 \leq k \leq N)$

$$
\Phi_{k}=\prod_{p=1}^{k} F_{p}
$$

Note: all $\Phi$ 's are functions on $[0, L]^{3 N}$ and $\Phi_{k}$ is independent of $x_{l}$ for $l>k$. We will choose $\Psi=\Phi_{N}$ for (2.1). 
As in [7, for proving the upper bound on the total energy $\left\langle\Phi_{N}\left|H_{N}\right| \Phi_{N}\right\rangle\left\|\Phi_{N}\right\|_{2}^{-2}$, we shall estimate the upper bounds on

$$
\left\|\Phi_{N}\right\|_{2}^{-2} \int \sum_{i}\left|\nabla_{i} \Phi_{N}\right|^{2} \prod_{j=1}^{N} d x_{j} \quad \text { and } \quad\left\|\Phi_{N}\right\|_{2}^{-2} \int \sum_{i<j} v^{a}\left(x_{i}-x_{j}\right)\left|\Phi_{N}\right|^{2} \prod_{k=1}^{N} d x_{k}
$$

Since in our case $v^{a}$ has negative parts, our strategy is more complicated, i.e., we need to estimate the upper bounds on

$$
\left\|\Phi_{N}\right\|_{2}^{-2} \int \sum_{i}\left|\nabla_{i} \Phi_{N}\right|^{2} \prod_{j=1}^{N} d x_{j} \quad \text { and } \quad\left\|\Phi_{N}\right\|_{2}^{-2} \int \sum_{i<j}\left[v^{a}\right]_{+}\left(x_{i}-x_{j}\right)\left|\Phi_{N}\right|^{2} \prod_{k=1}^{N} d x_{k}
$$

and the lower bound on

$$
\left\|\Phi_{N}\right\|_{2}^{-2} \int \sum_{i<j}\left|\left[v^{a}\right]_{-}\left(x_{i}-x_{j}\right)\right| \cdot\left|\Phi_{N}\right|^{2} \prod_{k=1}^{N} d x_{k}
$$

In the remainder of this section we are going to prove the following three inequalities

- $\left\|\Phi_{N}\right\|_{2}^{-2} \int \sum_{i}\left|\nabla_{i} \Phi_{N}\right|^{2} \prod_{j=1}^{N} d x_{j} \leq(1+o(1)) \frac{N^{2}}{\Lambda} \int_{\mathbb{R}^{3}}|\nabla f(x)|^{2} d x$

- $\left\|\Phi_{N}\right\|_{2}^{-2} \int \sum_{i<j}\left[v^{a}\right]_{+}\left(x_{i}-x_{j}\right)\left|\Phi_{N}\right|^{2} \prod_{k=1}^{N} d x_{k} \leq(1+o(1)) \frac{N^{2}}{\Lambda} \int_{\mathbb{R}^{3}} \frac{1}{2}[v]_{+}|f(x)|^{2} d x$

- $\left\|\Phi_{N}\right\|_{2}^{-2} \int \sum_{i<j}\left|\left[v^{a}\right]_{-}\left(x_{i}-x_{j}\right)\right| \cdot\left|\Phi_{N}\right|^{2} \prod_{k=1}^{N} d x_{k} \geq(1-o(1)) \frac{N^{2}}{\Lambda} \int_{\mathbb{R}^{3}} \frac{1}{2}\left|[v]_{-}\right| \cdot$ $|f(x)|^{2} d x$.

To prove these inequalities, we begin with proving the following three inequalities (all $\Phi$ 's are functions on $[0, L]^{3 N}$ ):

1. For any $m$-variable function $g_{m}\left(x_{i_{1}} \cdots x_{i_{m}}\right), m<k \leq N, i_{j} \neq i_{k}$ for $j \neq k$, we have

$$
\left\|\Phi_{k} F_{i_{1}}^{-1} \cdots F_{i_{m}}^{-1} g_{m}\right\|_{2}^{2} \leq(2 M)^{2 m} \Lambda^{-m}\left\|\Phi_{k-m}\right\|_{2}^{2}\left\|g_{m}\right\|_{2}^{2}
$$

2. For any two variable function $g_{2}\left(x_{i}, x_{i^{\prime}}\right)\left(i<i^{\prime}\right)$, we have

$$
\left\|\Phi_{N} F_{i^{\prime}}^{-1} g_{2}\right\|_{2}^{2} \leq\left\|g_{2}\right\|_{2}^{2} \Lambda^{-2}\left\|\Phi_{N}\right\|_{2}^{2}(1+\text { const. } Y)
$$

3. Let $f_{i, i^{\prime}}=f\left(x_{i}-x_{i^{\prime}}\right)$, for any two variable function $g_{2}\left(x_{i}, x_{i^{\prime}}\right)\left(i<i^{\prime}\right)$, we have

$$
\left\|\Phi_{N} f_{i, i^{\prime}}^{-1} g_{2}\right\|_{2}^{2} \geq\left\|g_{2}\right\|_{2}^{2} \Lambda^{-2}\left\|\Phi_{N}\right\|_{2}^{2}(1-\text { const. } Y)
$$

Note: if $f_{i, i^{\prime}}=0$ and $i<i^{\prime}$, then $F_{i^{\prime}}=0$, so $\Phi_{N} f_{i, i^{\prime}}^{-1}$ is definable.

We will use (2.22) for controlling the error terms. The inequalities (2.23) and (2.24) will be used in estimating the terms (2.20) and (2.21), respectively. 
We begin with deriving a lower bound on $\left\|\Phi_{N}\right\|_{2}^{2}$. For $i \neq p$, Let $F_{p, i}$ be the value that $F_{p}$ would take if changing the order of particles as follows,

$$
F_{p, i}\left(x_{1} \cdots x_{N}\right) \equiv F_{n(p, i)}\left(x_{1} \cdots x_{i-1}, x_{i+1} \cdots x_{N}, x_{i}\right)
$$

Here $n(p, i)$ is defined as follows $(i \neq p)$

$$
n(p, i)= \begin{cases}p & i>p \\ p-1 & i<p\end{cases}
$$

Similarly, we can define $F_{p, i, j}\left(x_{1} \cdots x_{N}\right)$ as

$$
F_{p, i, j}\left(x_{1} \cdots x_{N}\right) \equiv F_{m(p, i, j)}\left(x_{1} \cdots x_{i-1}, x_{i+1} \cdots x_{j-1}, x_{j+1} \cdots x_{N}, x_{i}, x_{j}\right) \text { for } i<j
$$

and $F_{p, i, j}=F_{p, j, i}$ for $j<i$. Here $m(p, i, j)$ is defined as the number of the elements of the set $\{1, \cdots, p\} \backslash\{i, j\}$.

Note: As we mentioned $F_{p}$ we defined is equal to the $F_{p}$ in [6] in the case when $v \geq 0$. Furthermore, one can see that our definitions of $F_{p, i}$ and $F_{p, i, j}$ are equivalent to those definitions in [6] when $v \geq 0$.

With the definitions of $F_{p}$ and $F_{p, i}$, we obtain that $F_{p, i}$ is independent of $x_{i}$ and $F_{p}$ is bounded from below as follows

$$
F_{p}\left(x_{1} \cdots x_{N}\right) \geq \begin{cases}F_{p, i} & i>p \\ F_{p, i} \bar{\theta}_{b}\left(x_{p}-x_{i}\right) & i<p\end{cases}
$$

and

$$
F_{p} \geq \prod_{i<p} \bar{\theta}_{b}\left(x_{p}-x_{i}\right)
$$

Then $\Phi_{N}^{2}$ is bounded from below, for any fixed $i$, by

$$
\begin{aligned}
\left|F_{1} \cdot F_{2} \cdots F_{N}\right|^{2} & \geq\left|F_{1, i} \cdots F_{i-1, i} F_{i+1, i} \cdots F_{N, i}\right|^{2} \times \prod_{j \neq i} \bar{\theta}_{b}\left(x_{i}-x_{j}\right) \\
& \geq\left|F_{1, i} \cdots F_{i-1, i} F_{i+1, i} \cdots F_{N, i}\right|^{2} \times\left(1-\sum_{j \neq i} \theta_{b}\left(x_{i}-x_{j}\right)\right)
\end{aligned}
$$

Integrating both sides with $\int \prod_{j=1}^{N} d x_{j}$, we obtain that

$$
\left\|\Phi_{N}\right\|_{2}^{2} \geq\left\|\Phi_{N-1}\right\|_{2}^{2}\left(1-\frac{4 \pi b^{3}}{3} N / \Lambda\right)=\left\|\Phi_{N-1}\right\|_{2}^{2}(1-Y)
$$

Here we used the fact that

$$
\left\|\Phi_{N-1}\right\|_{2}^{2}=\int\left|F_{1, i} \cdots F_{i-1, i} F_{i+1, i} \cdots F_{N, i}\right|^{2} \prod_{j} d x_{j} .
$$

Similarly, one can also prove that for $k \leq N$,

$$
\left\|\Phi_{k}\right\|_{2}^{2} \geq\left\|\Phi_{k-1}\right\|_{2}^{2}(1-Y)
$$


Next we are going to prove (2.22) in the case $m=1, k=N$, i.e.,

$$
\left\|\Phi_{N} F_{i}^{-1} g_{1}\right\|_{2}^{2} \leq 4 M^{2} \Lambda^{-1}\left\|\Phi_{N-1}\right\|_{2}^{2}\left\|g_{1}\right\|_{2}^{2}
$$

One can check that $F_{p}>F_{p, i}$ only when the following conditions are satisfied:

1. $i<p$

2. $\left|x_{i}-x_{p}\right| \leq \widetilde{R}$

3. for any other $j<p,\left|x_{j}-x_{p}\right|$ is greater than $\widetilde{R}$,

4. $T\left(R_{p}\right)<1$, i.e. for any other $j<p,\left|x_{j}-x_{p}\right|>2 \widetilde{R}$, i.e.,

$$
F_{p}>F_{p, i} \Rightarrow G_{p, i} \equiv \theta_{\widetilde{R}}\left(x_{p}-x_{i}\right) \prod_{j<p, j \neq i} \bar{\theta}_{2 \widetilde{R}}\left(x_{j}-x_{p}\right)=1
$$

On the other hand, using the fact that $f(\widetilde{R})>\frac{1}{2}$, one obtains that if $F_{p}>F_{p, i}$,

$$
F_{p, i} \prod_{j<p, j \neq i} \bar{\theta}_{2 \widetilde{R}}\left(x_{j}-x_{p}\right)>f(\widetilde{R}) \prod_{j<p, j \neq i} \bar{\theta}_{2 \widetilde{R}}\left(x_{j}-x_{p}\right) \geq \frac{1}{2} \prod_{j<p, j \neq i} \bar{\theta}_{2 \widetilde{R}}\left(x_{j}-x_{p}\right)
$$

Hence when $G_{p, i}=1$, we have $2 M F_{p, i} G_{p, i} \geq M \geq F_{p}$, i.e.,

$$
F_{p} \leq \begin{cases}F_{p, i} & i>p \\ F_{p, i}\left(1+(2 M-1) G_{p, i}\right) & i<p\end{cases}
$$

By the definition of $G^{\prime}$ 's, one can see that if $p, q>i$ and $p \neq q$,

$$
G_{p, i} G_{q, i}=0
$$

Hence, we have that

$$
\prod_{p>i}\left(1+(2 M-1) G_{p, i}\right) \leq 2 M
$$

Combining (2.35) and (2.37), we have the upper bound on $\left|\Phi_{N} F_{i}^{-1}\right|$ as follows,

$$
\left|F_{1} \cdot F_{2} \cdots F_{N} F_{i}^{-1}\right|^{2} \leq 4 M^{2}\left|F_{1, i} \cdots F_{i-1, i} F_{i+1, i} \cdots F_{N, i}\right|^{2},
$$

which implies the desired result (2.33) with (2.31). Furthermore, for any $m$-variable function $g_{m}\left(x_{i_{1}} \cdots x_{i_{m}}\right)$

$$
\left\|\Phi_{N} F_{i_{1}}^{-1} \cdots F_{i_{m}}^{-1} g_{m}\right\|_{2}^{2} \leq(2 M)^{2 m} \Lambda^{-m}\left\|\Phi_{N-m}\right\|_{2}^{2}\left\|g_{m}\right\|_{2}^{2}
$$


With the inequality (2.30) and the fact $F_{i} \leq M$ for any $i \leq N$, we get

$$
\begin{aligned}
\left\|\Phi_{N} g_{m}\right\|_{2}^{2} & \leq\left(2 M^{2}\right)^{2 m}\left\|\Phi_{N-m}\right\|_{2}^{2}\left\|g_{m}\right\|_{2}^{2} \\
& \leq(1-Y)^{-m}\left(2 M^{2}\right)^{2 m} \Lambda^{-m}\left\|\Phi_{N}\right\|_{2}^{2}\left\|g_{m}\right\|_{2}^{2}
\end{aligned}
$$

Similarly, we can generalize this result to $m<k \leq N$

$$
\begin{aligned}
\left\|\Phi_{k} g_{m}\right\|_{2}^{2} & \leq\left(2 M^{2}\right)^{2 m}\left\|\Phi_{k-m}\right\|_{2}^{2}\left\|g_{m}\right\|_{2}^{2} \\
& \leq(1-Y)^{-m}\left(2 M^{2}\right)^{2 m} \Lambda^{-m}\left\|\Phi_{k}\right\|_{2}^{2}\left\|g_{m}\right\|_{2}^{2}
\end{aligned}
$$

Now we shall prove the upper bound on $\left\|\Phi_{N}\right\|_{2}^{2}$ with (2.41). Choosing $p=N$, with the bounds of $F_{p}$ in (2.18), we get that

$$
\begin{aligned}
\Phi_{N}^{2} & \leq F_{1}^{2} \cdot F_{2}^{2} \cdots F_{N-1}^{2}\left(1+\sum_{j<N} M^{2} \theta_{\widetilde{R}}\left(x_{j}-x_{N}\right)\right) \\
& =\Phi_{N-1}^{2}+\Phi_{N-1}^{2} \sum_{j<N} M^{2} \theta_{\widetilde{R}}\left(x_{j}-x_{N}\right)
\end{aligned}
$$

Hence, using the inequalities (2.41) $(m=1)$ and (2.32), we obtain that

$$
\begin{aligned}
\left\|\Phi_{N}\right\|_{2}^{2} & \leq\left\|\Phi_{N-1}\right\|_{2}^{2}+(1-Y)^{-1}\left(2 M^{2}\right)^{2} Y\left\|\Phi_{N-2}\right\|_{2}^{2} \\
& \leq\left\|\Phi_{N-1}\right\|_{2}^{2}(1+\text { const. } Y)
\end{aligned}
$$

Putting (2.43) and (2.30) together, we obtain the relation between $\left\|\Phi_{N}\right\|$ and $\left\|\Phi_{N-1}\right\|$

$$
\left\|\Phi_{N}\right\|_{2}^{2}=\left\|\Phi_{N-1}\right\|_{2}^{2}(1+O(Y))
$$

Similarly, for $k \leq N$

$$
\left\|\Phi_{k}\right\|_{2}^{2}=\left\|\Phi_{k-1}\right\|_{2}^{2}(1+O(Y))
$$

Next, we shall prove (2.23), i.e.,

$$
\left\|\Phi_{N} F_{i^{\prime}}^{-1} g_{2}\right\|_{2}^{2} \leq\left\|g_{2}\right\|_{2}^{2} \Lambda^{-2}\left\|\Phi_{N}\right\|_{2}^{2}(1+\text { const. } Y) .
$$

Using the inequalities $F_{i} \leq\left(1+\sum_{l<i}(M-1) \theta_{\widetilde{R}}\left(x_{l}-x_{i}\right)\right)$ and (2.35), with the property of the $G$ 's in (2.36) $)$, we get

$$
\prod_{k \neq i^{\prime}} F_{k} \leq\left(1+\sum_{l<i}(M-1) \theta_{\widetilde{R}}\left(x_{l}-x_{i}\right)\right) \prod_{k \neq i^{\prime}, i} F_{k, i} \cdot\left(1+\sum_{j>i} 2 M G_{j, i}\right)(2
$$

Similarly, replacing $F_{p, i}$ 's with $F_{p, i, i^{\prime}}$ 's and using the fact that $G_{k, l} \leq \theta_{\widetilde{R}}\left(x_{k}-x_{l}\right)$, we get

$$
\begin{aligned}
\Phi_{N} F_{i^{\prime}}^{-1} \leq & \prod_{k \neq i^{\prime}, i} F_{k, i, i^{\prime}}\left(1+\sum_{l<i} M \theta_{\widetilde{R}}\left(x_{l}-x_{i}\right)\right) \\
& \times\left(1+\sum_{j>i} 2 M \theta_{\widetilde{R}}\left(x_{j}-x_{i}\right)\right) \times\left(1+\sum_{j^{\prime}>i^{\prime}} 2 M \theta_{\widetilde{R}}\left(x_{j}^{\prime}-x_{i}^{\prime}\right)\right)
\end{aligned}
$$


Expanding (2.48), multiplying $g_{2}\left(x_{i}, x_{i^{\prime}}\right)$ to each side and integrating them with $\prod_{k=1}^{N} d x_{k}$, with the result of (2.41, 2.45), we obtain that

$$
\left\|\Phi_{N} F_{i^{\prime}}^{-1} g_{2}\right\|_{2}^{2} \leq\left\|g_{2}\right\|_{2}^{2} \Lambda^{-2}\left\|\Phi_{N}\right\|_{2}^{2}(1+\text { const. } Y)
$$

So far we proved some upper bounds of the expectation value of $\Phi_{N}$. Next we are going to prove the following lower bound on $\left\|\Phi_{N} f_{i, i^{\prime}}^{-1} g_{2}\right\|_{2}^{2}$ :

$$
\left\|\Phi_{N} f_{i, i^{\prime}}^{-1} g_{2}\right\|_{2}^{2} \geq\left\|g_{2}\right\|_{2}^{2} \Lambda^{-2}\left\|\Phi_{N}\right\|_{2}^{2}(1-\text { const. } Y)
$$

Here we denote $f_{i, i^{\prime}}=f\left(x_{i}-x_{i^{\prime}}\right)\left(i<i^{\prime}\right)$. First, by the definition of $F_{i^{\prime}}$, one can see that $F_{i^{\prime}}=f\left(x_{i}-x_{i^{\prime}}\right)$ when $\prod_{k<i^{\prime}, k \neq i} \bar{\theta}_{b}\left(x_{k}-x_{i}^{\prime}\right)=1$, i.e.,

$$
F_{i^{\prime}}^{2} \geq f\left(x_{i}-x_{i^{\prime}}\right)^{2}\left(1-\sum_{k<i^{\prime}, k \neq i} \theta_{b}\left(x_{k}-x_{i^{\prime}}\right)\right)
$$

Using this inequality and (2.39) with $m=3, i_{1}=i, i_{2}=i^{\prime}$ and $i_{3}=k$, we obtain that

$$
\left\|\Phi_{N} f_{i, i^{\prime}}^{-1} g_{2}\right\|_{2}^{2} \geq\left\|\Phi_{N} F_{i^{\prime}}^{-1} g_{2}\right\|_{2}^{2}-\text { const. } Y\left\|g_{2}\right\|_{2}^{2} \Lambda^{-2}\left\|\Phi_{N}\right\|_{2}^{2}
$$

Then with the lower bound on $F_{i}^{2}$ in (2.18), i.e., $F_{i}^{2} \geq 1-\sum_{k<i} \theta_{b}\left(x_{k}-x_{i}\right)$, we obtain that

$$
\left\|\Phi_{N} f_{i, i^{\prime}}^{-1} g_{2}\right\|_{2}^{2} \geq\left\|\Phi_{N} F_{i}^{-1} F_{i^{\prime}}^{-1} g_{2}\right\|_{2}^{2}-\text { const. } Y\left\|g_{2}\right\|_{2}^{2} \Lambda^{-2}\left\|\Phi_{N}\right\|_{2}^{2}
$$

Again, using the bound on $F_{p}$ in (2.28), we see that

$$
\Phi_{N} F_{i}^{-1} F_{i^{\prime}}^{-1} \geq \prod_{k \neq i, i^{\prime}} F_{k, i, i^{\prime}}\left(1-\sum_{l<i} \theta_{b}\left(x_{l}-x_{i}\right)\right) \times\left(1-\sum_{l^{\prime}<i^{\prime}, l^{\prime} \neq i} \theta_{b}\left(x_{l^{\prime}}-x_{i^{\prime}}\right)\right)
$$

Then using (2.41) and (2.45), we arrive at the desired result (2.50).

So far, we have proved the inequalities we need for calculating the value of $\left\langle\Phi_{N}\left|\sum_{i, j} v^{a}\left(x_{i}-x_{j}\right)\right| \Phi_{N}\right\rangle$. Then we need to calculate $\nabla_{i} \Phi_{N}$. We denote $i_{p}$ as the particle satisfying $i_{p}<p$ and $\left|x_{i_{p}}-x_{p}\right|=r_{p}$ and $n_{p}^{r}$ as the unit vector in the direction of $x_{p}-x_{i_{p}}$. Similarly, denote $j_{p}$ as the particle satisfying $j_{p}<p$ and $\left|x_{j_{p}}-x_{p}\right|=R_{p}$ and $n_{p}^{R}$ as the unit vector in the direction of $x_{p}-x_{j_{p}}$. We remark that such $i_{p}$ or $j_{p}$ may not exist in some cases, but we do define them as 0 . We denote $\nabla_{0} F_{p}=0$. Recall the definition of $F_{p}$ in (2.13). We have

$$
\begin{aligned}
-\nabla_{p} F_{p} & =\nabla_{i_{p}} F_{p}+\nabla_{j_{p}} F_{p} \\
\nabla_{i_{p}} F_{p} & =-n_{p}^{r} f^{\prime}\left(r_{p}\right)\left(\Theta_{p}^{i n}+\Theta_{p}^{-}\left(1-T\left(R_{p}\right)\right)+\Theta_{p}^{+}\right) \\
\nabla_{j_{p}} F_{p} & =-n_{p}^{R}\left(\Theta_{p}^{\text {out }} f^{\prime}\left(R_{p}\right)+\Theta_{p}^{-} T\left(R_{p}\right) f^{\prime}\left(R_{p}\right)+\Theta_{p}^{-} T^{\prime}\left(R_{p}\right)\left(f\left(R_{p}\right)-f\left(r_{p}\right)\right)\right)
\end{aligned}
$$


Here $\Theta_{p}^{+}$is the function of $\left(x_{1} \cdots x_{N}\right)$ which is defined as

$$
\Theta_{p}^{+} \equiv\left[1-\Theta^{\text {in }}-\Theta^{\text {out }}\right] \cdot h\left[f\left(R_{p}\right)-f\left(r_{p}\right)\right]
$$

and $\Theta_{p}^{-}$is defined as

$$
\Theta_{p}^{-} \equiv\left[1-\Theta^{\text {in }}-\Theta^{\text {out }}\right] \cdot h\left[f\left(r_{p}\right)-f\left(R_{p}\right)\right]
$$

Here $h$ is the Heaviside step function. By the definition of $\Phi_{N}$, we obtain that

$$
\frac{\left|\nabla_{p} \Phi_{N}\right|^{2}}{\left|\Phi_{N}\right|^{2}}=\left|-F_{p}^{-1} \nabla_{i_{p}} F_{p}-F_{p}^{-1} \nabla_{j_{p}} F_{p}+\sum_{q, i_{q}=p} F_{q}^{-1} \nabla_{p} F_{q}+\sum_{q, j_{q}=p} F_{q}^{-1} \nabla_{p} F_{q}\right|^{2}
$$

Then with (2.54), we have that

$$
\begin{aligned}
\sum_{p}\left|\nabla_{p} \Phi_{N}\right|^{2} \leq \quad & 2\left|\Phi_{N}\right|^{2} \sum_{p} F_{p}^{-2}\left(\left|f^{\prime}\left(r_{p}\right)\right|^{2}\left(\Theta_{p}^{i n}+\Theta_{p}^{-}\left|1-T\left(R_{p}\right)\right|^{2}+\Theta_{p}^{+}\right)\right. \\
& +\left|T\left(R_{p}\right) f^{\prime}\left(R_{p}\right)\right|^{2} \Theta_{p}^{-}+\left|T^{\prime}\left(R_{p}\right)\right|^{2}\left|f\left(R_{p}\right)-f\left(r_{p}\right)\right|^{2} \Theta_{p}^{-} \\
& \left.+\left|T\left(R_{p}\right)\right| \cdot\left|1-T\left(R_{p}\right)\right| \cdot\left|f^{\prime}\left(r_{p}\right)\right| \cdot\left|f^{\prime}\left(R_{p}\right)\right| \Theta_{p}^{-}+\left|f^{\prime}\left(R_{p}\right)\right|^{2} \Theta_{p}^{\text {out }}\right) \\
& +2\left|\Phi_{N}\right|^{2} \sum_{k<p<q} F_{p}^{-1} F_{q}^{-1}\left(\left|\nabla_{k} F_{p}\right| \cdot\left|\nabla_{p} F_{q}\right|+\left|\nabla_{k} F_{p}\right| \cdot\left|\nabla_{k} F_{q}\right|\right)
\end{aligned}
$$

Because $0 \leq T \leq 1$ and $i_{p} \neq j_{p}$, one can easily prove that for any fixed $p$,

$$
\begin{aligned}
& \left(\left|f^{\prime}\left(r_{p}\right)\right|^{2}\left(\Theta_{p}^{\text {in }}+\Theta_{p}^{-}\left|1-T\left(R_{p}\right)\right|^{2}+\Theta_{p}^{+}\right)+\left|f^{\prime}\left(R_{p}\right)\right|^{2} \Theta_{p}^{\text {out }}\right. \\
+\quad & \left.\left|T\left(R_{p}\right) f^{\prime}\left(R_{p}\right)\right|^{2} \Theta_{p}^{-}+\left|T\left(R_{p}\right)\right| \cdot\left|1-T\left(R_{p}\right)\right| \cdot\left|f^{\prime}\left(r_{p}\right)\right| \cdot\left|f^{\prime}\left(R_{p}\right)\right| \Theta_{p}^{-}\right) \\
\leq & \sum_{k: k<p} f^{\prime}\left(\left|x_{p}-x_{k}\right|\right)^{2},
\end{aligned}
$$

and

$$
\left|T^{\prime}\left(R_{p}\right)\right|^{2}\left|f\left(R_{p}\right)-f\left(r_{p}\right)\right|^{2} \Theta_{p}^{-} \leq M^{2} \sum_{k: k<p}\left(T^{\prime}\left(\left|x_{p}-x_{k}\right|\right)^{2} \sum_{j: j \neq k, p} \theta_{\widetilde{R}}\left(x_{j}-x_{p}\right)\right)
$$


Hence, we obtain that

$$
\begin{aligned}
& \left\langle\Phi_{N}\left|H_{N}\right| \Phi_{N}\right\rangle \leq 2 \sum_{i<j} \int\left|\Phi_{N}\right|^{2} F_{j}^{-2}\left(\frac{1}{2} f\left(x_{i}-x_{j}\right)^{2}\left[v\left(x_{i}-x_{j}\right)\right]_{+}+f^{\prime}\left(x_{i}-x_{j}\right)^{2}\right) \\
& -2 \sum_{i<j} \int\left|\Phi_{N}\right|^{2} f^{-2}\left(x_{i}-x_{j}\right)\left(\frac{1}{2} f^{2}\left(x_{i}-x_{j}\right)\left|\left[v\left(x_{i}-x_{j}\right)\right]_{-}\right|\right) \\
& +2 \sum_{i<j} \int\left|\Phi_{N}\right|^{2} M^{2} \sum_{k<p}\left(T^{\prime}\left(\left|x_{p}-x_{k}\right|\right)^{2} \sum_{j: j \neq k, p} \theta_{\widetilde{R}}\left(x_{j}-x_{p}\right)\right) \\
& +2 \sum_{k<p<q} \int\left|\Phi_{N}\right|^{2} F_{p}^{-1} F_{q}^{-1}\left(\left|\nabla_{k} F_{p}\right| \cdot\left|\nabla_{p} F_{q}\right|+\left|\nabla_{k} F_{p}\right| \cdot\left|\nabla_{k} F_{q}\right|\right)
\end{aligned}
$$

Here $[\cdot]_{+}$and $[\cdot]_{-}$denote the positive and negative part, respectively and we used the fact that $F_{j} \leq f\left(x_{i}-x_{j}\right)$ when $i<j$ and $\left|x_{i}-x_{j}\right| \leq \widetilde{R}$, which implies that

$$
\left[v\left(x_{i}-x_{j}\right)\right]_{+} \leq F_{j}^{-2} f\left(x_{i}-x_{j}\right)^{2}\left[v\left(x_{i}-x_{j}\right)\right]_{+}
$$

With the results in (2.49) and (2.50), we can obtain the upper bound on the main part of $\left\langle\Phi_{N}\left|H_{N}\right| \Phi_{N}\right\rangle$, i.e.,

$$
\begin{aligned}
& 2 \sum_{i<j} \int\left|\Phi_{N}\right|^{2} \times \\
& \frac{1}{2}\left(F_{j}^{-2}\left[f^{2}\left(x_{i}-x_{j}\right)\left[v\left(x_{i}-x_{j}\right)\right]_{+}+f^{\prime}\left(x_{i}-x_{j}\right)^{2}\right]-\frac{f^{2}\left(x_{i}-x_{j}\right)}{f^{2}\left(x_{i}-x_{j}\right)}\left|\left[v\left(x_{i}-x_{j}\right)\right]_{-}\right|\right) \\
& \leq 4 \pi a N^{2} / \Lambda(1+\text { const. } Y)\left\|\Phi_{N}\right\|_{2}^{2}
\end{aligned}
$$

With the definition of $T$ in (2.12) and (2.40), we obtain that the third line of (2.58) is bounded as const. $a N^{2} Y\left\|\Phi_{N}\right\|_{2}^{2} / \Lambda$. For the other terms, we have

$$
\left|\nabla_{i_{p}} F_{p}\right| \leq\left|f^{\prime}\left(\left|x_{i_{p}}-x_{p}\right|\right)\right|, \quad\left|\nabla_{j_{p}} F_{p}\right| \leq\left|f^{\prime}\left(\left|x_{j_{p}}-x_{p}\right|\right)\right|+M T^{\prime}\left(\left|x_{j_{p}}-x_{p}\right|\right)
$$

Hence, with the inequality (2.39), we can prove that the last line in (2.58) are bounded as

$$
\text { const. } N^{3} \Lambda^{-2}(K+L)^{2}\left\|\Phi_{N}\right\|_{2}^{2}
$$

Here $K$ and $L$ are defined as follows

$$
K \equiv \int_{\mathbb{R}^{3}}\left|f^{\prime}(|x-y|)\right| d y \quad L \equiv \int_{\mathbb{R}^{3}} T^{\prime}(|x-y|) d y .
$$

Note that $K$ and $L$ are independent of $x$. By the definitions of $f$ in (2.8) and $T$ in (2.12), we get that

$$
K=O(a b), \quad L=O(\widetilde{R} b)=O(a b)
$$

Hence we obtain that the last line in (2.58) are bounded by const. $a N^{2} Y^{2}$. Combining this result with (2.60), we get the following result,

$$
\frac{\left\langle\Phi_{N}\left|H_{N}\right| \Phi_{N}\right\rangle}{\left\|\Phi_{N}\right\|_{2}^{2}} \leq 4 \pi a N^{2} / \Lambda(1+\text { const. } Y)
$$


At last, by choosing $\Psi=\Phi_{N}$, we arrive at the desired result (2.1), which implies Theorem 1.

\subsection{Proof of Theorem Two}

Proof. Following the ideas in [7], we need to replace the hard potential by a soft potential at the expense of local kinetic energy. This method has been used in many papers on dilute bose or fermi gases $[7,6,6,2,3]$. But in this method the kinetic energy of particle $i$ only can be used for the hard-soft potential replacement between the particle $i$ and one other $j$ (the nearest particle [7]). In our case that $v^{a}$ is partly negative, we can not ignore the potential between $i$ and other $k$ 's for the lower bound on the energy. To solve this problem, we begin with separating the whole Hamiltonian into two parts, (1) The Hamiltonian of the energy when two particles are close to each other and they are far away from the others. (2) The Hamiltonian of the remaining energy. In the remainder of this section, we prove that the first part is greater than $4 \pi a N^{2} \Lambda^{-1}\left(1-O\left(a^{3} \varrho\right)^{1 / 17}\right)$ and the second part is non-negative.

Another important property Lieb and Yngvason used in [7] is the superadditivity of the ground energy $E(n, \ell)$ of $n$ particles in $[0, \ell]^{3}$ with Neumann boundary condition, i.e.,

$$
E\left(n+n^{\prime}, \ell\right) \geq E(n, \ell)+E\left(n^{\prime}, \ell\right)
$$

This property is trivial in the case $v^{a} \geq 0$. In our proof, we are not going to prove any similar property, actually we only need the property (2.134) that for fixed $\ell$, when $n$ is larger than $4 \varrho \ell^{3}$, the energy/particle is greater than $8 \pi a \varrho$, as in $(2.62)$ of [5], i.e.,

$$
E(n, \ell) / n \geq 8 \pi a \varrho\left(1-\text { const. }\left(a^{3} \varrho\right)^{1 / 17}\right)
$$

which will be proved in Lemma 1.

Choosing

$$
R=a\left(a^{3} \varrho\right)^{-5 / 17} \geq 2 R_{0} a,
$$

we define $F_{i, j}$ for $i \neq j$ as follows:

$$
F_{i, j}=\theta_{R}\left(x_{i}-x_{j}\right) \prod_{k \neq i, j} \bar{\theta}_{2 R}\left(x_{i}-x_{k}\right)
$$

Here $\theta_{R}$ is the characteristic function of the open set $|x|<R$, and $\bar{\theta}_{R}=1-\theta_{R}$. We note that $F_{i, j} \neq F_{j, i}$ and $F_{i, j}$ is equal to 1 only when $x_{j}$ is close to $x_{i}$, but the other $x_{k}$ 's are not. It is easy to check that $\sum_{i: i \neq j} F_{i, j} \leq 1$, so

$$
-\nabla_{j} \sum_{i: i \neq j} F_{i, j} \nabla_{j} \leq-\Delta_{j}
$$

for any fixed $x_{1}, \cdots x_{j-1}, x_{j+1}, \cdots, x_{N}$.

Then we denote $v_{+}^{a}$ and $v_{-}^{a}$ as scaled potentials as follows,

$$
v_{+}^{a}(r)=a^{-2} v_{+}(r / a), \quad v_{-}^{a}(r)=a^{-2} v_{-}(r / a)
$$


Choosing

$$
Y=\left(a^{3} \varrho\right)^{1 / 17}
$$

and $\varepsilon$ satisfying

$$
3 \cdot\left(\min \left\{1, S L\left[v_{+}\right]\right\}\right)^{-1} \cdot Y=\varepsilon<\frac{t}{2(1+t)}
$$

with the definition

$$
v_{\varepsilon}^{a} \equiv v^{a}-\varepsilon v_{+}^{a},
$$

we separate the Hamiltonian $H_{N}$ as follows

$$
\begin{aligned}
& H_{N}= \\
& (1-\varepsilon) \sum_{j}-\nabla_{j} \sum_{i} F_{i, j} \nabla_{j}+\sum_{i \neq j} F_{i j} \frac{v_{\varepsilon}^{a}}{2}\left(x_{i}-x_{j}\right)-\varepsilon \sum_{j} \Delta_{j}+\varepsilon \sum_{i \neq j} \frac{v_{+}^{a}}{2}\left(x_{i}-x_{j}\right) \\
& +(1-\varepsilon) \sum_{j}-\nabla_{j}\left(1-\sum_{i} F_{i, j}\right) \nabla_{j}+\sum_{i \neq j}\left(1-F_{i j}\right) \frac{v_{\varepsilon}^{a}}{2}\left(x_{i}-x_{j}\right)
\end{aligned}
$$

First, we claim the following Lemma 1, which will be proved in next section.

Lemma 1. Define $Y, F_{i, j}, \varepsilon, v_{\varepsilon}^{a}$ and $R$ as in 2.72, (2.69), 2.73, 2.74 and (2.68) respectively. There exists $C$ depending only on $v$ such that

$$
\begin{aligned}
& H^{\prime \prime} \equiv \\
& (1-\varepsilon) \sum_{j}-\nabla_{j} \sum_{i} F_{i, j} \nabla_{j}+\sum_{i \neq j} F_{i j} \frac{v_{\varepsilon}^{a}}{2}\left(x_{i}-x_{j}\right)-\varepsilon \sum_{j} \Delta_{j}+\varepsilon \sum_{i \neq j} \frac{v_{+}^{a}}{2}\left(x_{i}-x_{j}\right) \\
& \geq 4 \pi a N^{2} / \Lambda(1-C Y)
\end{aligned}
$$

Hence, to obtain Theorem 2, it only remains to prove that the last line of (2.75), as an operator, is bounded from below by zero, i.e.,

$$
(1-\varepsilon) \sum_{j}-\nabla_{j}\left(1-\sum_{i} F_{i, j}\right) \nabla_{j}+\sum_{i \neq j}\left(1-F_{i j}\right) \frac{1}{2} v_{\varepsilon}^{a}\left(x_{i}-x_{j}\right) \geq 0
$$

By the assumptions $\varepsilon<t(2+2 t)^{-1}$, we have

$$
v_{\varepsilon}^{a} \geq \frac{2+t}{2+2 t} v_{+}^{a}+v_{-}^{a} .
$$

Hence, it remains to prove that

$$
0 \leq H_{N}^{\prime} \equiv \frac{2+t}{2+2 t} \sum_{j}-\nabla_{j}\left(1-\sum_{i} F_{i, j}\right) \nabla_{j}+\frac{1}{2} \sum_{i \neq j}\left(1-F_{i j}\right)\left(\frac{2+t}{2+2 t} v_{+}^{a}+v_{-}^{a}\right)\left(x_{i}-x_{j}\right)
$$


Because $\lim _{N \rightarrow \infty} E(N, \Lambda) / N$ exists, for proving Theorem 2, we can assume that $N$ is even, i.e., $N=2 N_{1}$. Consider any partition $P=\left(\pi_{1}, \pi_{2}\right)$ of $1, \ldots, N$ into two disjoint sets with $N_{1}$ integers in $\pi_{1}$ and $\pi_{2}$ respectively. For each $P$, we define that

$$
\begin{aligned}
H_{P}=H_{\left(\pi_{1}, \pi_{2}\right)} & \equiv \quad \frac{2+t}{1+t} \sum_{j \in \pi_{1}}-\nabla_{j}\left(1-\sum_{i \neq j} F_{i, j}\right) \nabla_{j}+\sum_{i, j \in \pi_{1}}\left(1-F_{i, j}\right) \frac{1}{2} v_{1,1}^{a}\left(x_{i}-x_{j}\right) \\
& +\quad \sum_{i \in \pi_{2}, j \in \pi_{1}}\left(1-F_{i, j}\right) \frac{1}{2} v_{2,1}^{a}\left(x_{i}-x_{j}\right)+\sum_{i, j \in \pi_{2}}\left(1-F_{i, j}\right) \frac{1}{2} v_{2,2}^{a}\left(x_{i}-x_{j}\right)
\end{aligned}
$$

Here we denote $v_{\alpha, \beta}^{a}$ as the interaction potential between particles in $\pi_{\alpha}$ and $\pi_{\beta}$, which are chosen as

$$
v_{1,1}^{a}=v_{2,2}^{a}=\frac{t}{1+t} v_{+}^{a} \geq 0, \quad v_{2,1}^{a}=\frac{4}{1+t} v_{+}^{a}+4 v_{-}^{a},
$$

so

$$
\frac{1}{4}\left(v_{1,1,}^{a}+v_{2,1}^{a}+v_{2,2}^{a}\right)=\frac{2+t}{2+2 t} v_{+}^{a}+v_{-}^{a} \leq v_{\varepsilon}^{a} .
$$

It is easily to check that

$$
H_{N}^{\prime}=\sum_{P} H_{P} / \sum_{P} 1
$$

Hence, to obtain $H_{N}^{\prime} \geq 0$, it remains to prove that for $\forall P, H_{P} \geq 0$. Because there is no kinetic energy of particles in $\pi_{2}$, we can fix the configuration of $x_{i}$ 's with $i \in \pi_{2}$. Since permutation of the labels in $\pi_{1}$ and $\pi_{2}$ is irrelevant, we assume that $\pi_{1}=\left\{1, \cdots, N_{1}\right\}, \pi_{2}=\left\{N_{1}+1, \cdots, N\right\}$.

As we can see $v_{2,1}^{a}$ is the only partly negative component in $H_{P}$. For fixed $\pi_{2}$ particles, we can write $v_{2,1}^{a}\left(x_{j}-x_{i}\right)$ as

$$
v_{2,1}^{a}\left(x_{j}-x_{i}\right)=v_{2,1}^{a}\left(x_{j}-x_{i}\right)\left(1-\chi_{A}\left(x_{i}\right)\right)+v_{2,1}^{a}\left(x_{j}-x_{i}\right) \chi_{A}\left(x_{i}\right)
$$

Here $\chi_{A}$ is the characteristic function of $A$, which is a subset of $[0, L]^{3}(2.96)$. We shall show $A$ is the area where the density of $\pi_{2}$ particles is less than some fixed number. To obtain $H_{P} \geq 0$, our strategy is to prove that

1. The total energy of the interaction potential $v_{1,1}^{a}$ and $v_{2,2}^{a}$ cancels out the negative part of $v_{2,1}^{a}\left(1-\chi_{A}\right)$.

2. The total kinetic energy and the positive part of $v_{2,1}^{a}$ cancels out the negative part of $v_{2,1}^{a} \chi_{A}$.

To make the strategy more clear, we shall define $A$ where the density of $\pi_{2}$ particles is less than some fixed number. First we divide the cubic box $[0, L]^{3}$ into small cubes $B_{n}(n \in \mathbb{N})$ of side length $\ell$, with

$$
\ell=\frac{1}{2} r_{1} a .
$$

Then, with fixed $x_{i}$ 's, $i \in \pi_{2}$, for any $x \in[0, L]^{3}$, we define the $G(x)$ as the set of $i$ 's which satisfy $i \in \pi_{2}$ and $\left|x_{i}-x\right| \leq R_{0} a$, i.e.,

$$
G(x) \equiv\left\{i \in \pi_{2}:\left|x_{i}-x\right| \leq R_{0} a\right\}
$$


We denote $|G(x)|$ as the number of the elements of $G(x)$.

We denote $d\left(x, B_{n}\right)$ as the distance between the cube $B_{n} \subset \mathbb{R}^{3}$ and $x \in \mathbb{R}^{3}$. Since $|G(y)|$ is uniformly bounded $\left(|G(y)| \leq N_{1}\right)$, there must exist a point $X\left(B_{n}\right) \in \mathbb{R}^{3}$ satisfying $d\left(X\left(B_{n}\right), B_{n}\right) \leq 2 R_{0} a$ and

$$
\left|G\left(X\left(B_{n}\right)\right)\right|=\max \left\{|G(y)|: d\left(y, B_{n}\right) \leq 2 R_{0} a\right\}
$$

We define $G\left(B_{n}\right) \equiv G\left(X\left(B_{n}\right)\right)$. We are going to prove that there exists $n_{1} \in \mathbb{N}$ depending on $R_{0} / r_{1}$ such that

1. The total energy of the interaction potential $v_{1,1}^{a}$ and $v_{2,2}^{a}$ cancels out the negative parts of $v_{2,1}^{a}\left(x_{j}, x_{i}\right)$ 's when $x_{i}$ is in a cube $B_{n}$ such that $\left|G\left(B_{n}\right)\right|>n_{1}$.

2. The total kinetic energy and the positive part of $v_{2,1}^{a}$ cancel out the negative part of the remaining $v_{2,1}^{a}$ 's.

First, we derive the lower bound on the total energy of $v_{2,2}^{a}$, i.e. (2.86, 2.88). With the definition of $G\left(B_{n}\right)=G\left(X\left(B_{n}\right)\right)$, we know that the set $\left\{x_{k}: k \in G\left(B_{n}\right)\right\}$ can be covered by a sphere of radius $R_{0} a$. So the number of the cubes which one need to cover this set is less than const. $\left(R_{0} / r_{1}\right)^{3}$. We denote these cubes as $B_{n_{1}} \cdots B_{n_{m}}$ ( $m \leq$ const. $\left.\left(R_{0} / r_{1}\right)^{3}\right)$ and assume the number of $i$ 's satisfying $i \in G\left(B_{n}\right)$ and $x_{i} \in$ $B_{n_{k}}$ is $a_{n_{k}}$. Because the side length of $B_{n_{k}}$ is equal to $r_{1} a / 2$, the distance between the two particles in the same cube is no more than $\frac{\sqrt{3}}{2} r_{1} a<r_{1} a$. Hence we have

$$
\begin{aligned}
\sum_{i, j \in G\left(B_{n}\right)} \theta_{r_{1} a}\left(x_{i}-x_{j}\right) & \geq \sum_{k=1}^{m}\left[\left(a_{n_{k}}\right)^{2}-\left(a_{n_{k}}\right)\right] \\
& \geq \frac{\left(\sum_{k=1}^{m} a_{n_{k}}\right)^{2}}{m}-\left(\sum_{k=1}^{m} a_{n_{k}}\right) \\
& \geq \operatorname{const.}\left(R_{0} / r_{1}\right)^{-3}\left|G\left(B_{n}\right)\right|^{2}-\left|G\left(B_{n}\right)\right|
\end{aligned}
$$

Hence, we obtain that there exist $n_{1} \geq 3$ and $n_{1}, n_{2}=$ const. $\left(R_{0} / r_{1}\right)^{3}$ such that when $\left|G\left(B_{n}\right)\right| \geq n_{1}$,

$$
\sum_{i, j \in G\left(B_{n}\right)} \theta_{r_{1} a}\left(x_{i}-x_{j}\right) \geq \frac{1}{n_{2}}\left|G\left(B_{n}\right)\right|^{2},
$$

which implies

$$
\sum_{i, j \in G\left(B_{n}\right)} v_{2,2}^{a}\left(x_{i}-x_{j}\right) \geq \frac{t \lambda_{+} a^{-2}}{(1+t) n_{2}}\left|G\left(B_{n}\right)\right|^{2}
$$

Here, we used (2.77) and (2.71), i.e.,

$$
v_{2,2}^{a}(r)=\frac{t}{(1+t)} v_{+}^{a}(r)=\frac{t}{(1+t)} a^{-2} v_{+}(r / a)
$$


Again, with the fact that the set $\left\{x_{k}: k \in G\left(B_{n}\right)\right\}$ can be covered with a sphere of diameter $2 R_{0} a \leq R$, one can see that if $i \in G\left(B_{n}\right)$ and $\left|G\left(B_{n}\right)\right| \geq 3$, we have $F_{i, j}=0$ for any $j \neq i$. Hence we obtain that, for any fixed $B_{n}$ satisfying $\left|G\left(B_{n}\right)\right| \geq n_{1}$,

$$
\sum_{i, j \in G\left(B_{n}\right)}\left(1-F_{i, j}\right) v_{2,2}^{a}\left(x_{i}-x_{j}\right)=\sum_{i, j \in G\left(B_{n}\right)} v_{2,2}^{a}\left(x_{i}-x_{j}\right) \geq \frac{t \lambda_{+} a^{-2}}{(1+t) n_{2}}\left|G\left(B_{n}\right)\right|^{2}
$$

Then, we are going to sum up all the cubes satisfying $\left|G\left(B_{n}\right)\right| \geq n_{1}$. It is easy to see that

$$
d\left(x_{i}, B_{n}\right) \leq 3 R_{0} a, \text { for } i \in G\left(B_{n}\right),
$$

which implies that for any fixed $i \in \pi_{2}$, the number of cubes $B_{n}$ 's satisfying $i \in G\left(B_{n}\right)$ is less than some constant $n_{3}$, which is less than const. $\left(R_{0} / r_{1}\right)^{3}$. Hence, summing up all the blocks satisfying $\left|G\left(B_{n}\right)\right| \geq n_{1}$, with the inequality (2.86), we get that

$$
\begin{aligned}
\sum_{i, j \in \pi_{2}}\left(1-F_{i, j}\right) v_{2,2}^{a}\left(x_{i}-x_{j}\right) & \geq \sum_{n:\left|G\left(B_{n}\right)\right| \geq n_{1}} \sum_{i, j \in G\left(B_{n}\right)}\left(1-F_{i, j}\right) v_{2,2}^{a}\left(x_{i}-x_{j}\right) \\
& \geq \sum_{n:\left|G\left(B_{n}\right)\right| \geq n_{1}} \frac{t \lambda_{+} a^{-2}}{(1+t) n_{2} n_{3}}\left|G\left(B_{n}\right)\right|^{2}
\end{aligned}
$$

Second, we derive the lower bound on the interaction potential between particles in $\pi_{1}$. Because the distance between any two points in the same cube is less than $r_{1} a$, we have $v_{1,1}^{a}\left(x_{i}-x_{j}\right) \geq a^{-2} \lambda_{+} t(1+t)^{-1}$ when $i, j \in \pi_{1}$ and $x_{i}, x_{j} \in B_{n}$, i.e.,

$$
\sum_{i, j \in \Pi_{1}\left(B_{n}\right)} v_{1,1}^{a}\left(x_{i}-x_{j}\right) \geq \frac{a^{-2} t \lambda_{+}}{1+t}\left(\left|\Pi_{1}\left(B_{n}\right)\right|^{2}-\left|\Pi_{1}\left(B_{n}\right)\right|\right)
$$

Here $\Pi_{1}\left(B_{n}\right)$ is defined as the set of $i$ 's such that $i \in \pi_{1}$ and $x_{i} \in B_{n}$ and $\left|\Pi_{1}\left(B_{n}\right)\right|$ is the number of the elements of $\Pi_{1}\left(B_{n}\right)$. Furthermore, if $x_{i} \in B_{n}$ and $\left|G\left(B_{n}\right)\right| \geq 1$, there must be a $k \in \pi_{2}$ satisfying $\left|x_{i}-x_{k}\right| \leq 4 R_{0} a \leq 2 R$, hence $F_{i, j}=0$ for any other $j \in \pi_{1}$. Using this result, for any $B_{n}$ satisfying $\left|G\left(B_{n}\right)\right| \geq 1$, we have that

$$
\sum_{i, j \in \Pi_{1}\left(B_{n}\right)}\left(1-F_{i, j}\right) v_{1,1}^{a}\left(x_{i}-x_{j}\right) \geq \frac{t \lambda_{+} a^{-2}}{1+t}\left(\left|\Pi_{1}\left(B_{n}\right)\right|^{2}-\left|\Pi_{1}\left(B_{n}\right)\right|\right)
$$

At last, we derive the lower bound on $v_{2,1}^{a}$. By the definitions of $\left|G\left(B_{n}\right)\right|$ and $v_{2,1}^{a}$, we have that $\forall x \in B_{n}$,

$$
\sum_{i \in \pi_{2}}\left[v_{2,1}^{a}\right]_{-}\left(x-x_{i}\right) \geq-4 \lambda_{-} a^{-2}\left|G\left(B_{n}\right)\right| .
$$

Here we denote $\left[v_{2,1}^{a}\right]_{-}$as the negative part of $v_{2,1}^{a}$ which is equal to $4\left[v^{a}\right]_{-}$. With the facts $0 \geq 4\left[v^{a}\right]_{-} \geq-4 \lambda_{-} a^{-2}$ and $0 \leq F_{i, j} \leq 1$, we have the following inequality

$$
\sum_{j \in \Pi_{1}\left(B_{n}\right), i \in \pi_{2}}\left(1-F_{i, j}\right)\left[v_{2,1}^{a}\right]_{-}\left(x_{i}-x_{j}\right) \geq-4 \lambda_{-} a^{-2} \cdot\left|\Pi_{1}\left(B_{n}\right)\right| \cdot\left|G\left(B_{n}\right)\right|
$$


One can check that if $\left|G\left(B_{n}\right)\right| \geq n_{1}$ and

$$
\lambda_{+} \geq\left(1+t^{-1}\right) \lambda_{-} \cdot \max \left\{2 \sqrt{n_{2} n_{3}}, \frac{n_{2} n_{3}}{4 n_{1}}\right\} \sim \text { const. }\left(1+t^{-1}\right) \lambda_{-}\left(R_{0} / r_{1}\right)^{3},
$$

the sum of the right sides of (2.90) and (2.91) is bounded from below as follows,

$$
\begin{aligned}
& -4 \lambda_{-} \cdot\left|\Pi_{1}\left(B_{n}\right)\right| \cdot\left|G\left(B_{n}\right)\right|+\frac{t \lambda_{+}}{1+t}\left(\left|\Pi_{1}\left(B_{n}\right)\right|^{2}-\left|\Pi_{1}\left(B_{n}\right)\right|\right) \\
\geq & -\frac{t \lambda_{+}}{(1+t) n_{2} n_{3}}\left|G\left(B_{n}\right)\right|^{2}
\end{aligned}
$$

Hence, with (2.90) and (2.91), we obtain that if (2.92) holds and $\left|G\left(B_{n}\right)\right| \geq n_{1}$,

$$
\begin{aligned}
0 & \leq \quad \frac{t}{1+t} \frac{\lambda_{+} a^{-2}}{n_{2} n_{3}}\left|G\left(B_{n}\right)\right|^{2}+\sum_{i, j \in \Pi_{1}\left(B_{n}\right)}\left(1-F_{i, j}\right) v_{1,1}^{a}\left(x_{i}-x_{j}\right) \\
& +\sum_{j \in \Pi_{1}\left(B_{n}\right), i \in \pi_{2}}\left(1-F_{i, j}\right)\left[v_{2,1}^{a}\right]_{-}\left(x_{i}-x_{j}\right),
\end{aligned}
$$

Then summing up all the $B_{n}$ 's satisfying $\left|G\left(B_{n}\right)\right|>n_{1}$, with (2.88) and $v_{11} \geq 0$, we obtain that as long as (2.92) holds,

$$
\begin{aligned}
0 \leq & \sum_{i, j \in \pi_{2}}\left(1-F_{i, j}\right) \frac{1}{2} v_{2,2}^{a}\left(x_{i}-x_{j}\right)+\sum_{i, j \in \pi_{1}}\left(1-F_{i, j}\right) \frac{1}{2} v_{1,1}^{a}\left(x_{i}-x_{j}\right) \\
& +\sum_{j \in \pi_{1}, i \in \pi_{2}}\left(1-F_{i, j}\right) \frac{1}{2}\left[v_{2,1}^{a}\right]_{-}\left(x_{i}-x_{j}\right)\left(1-\chi_{A}\left(x_{j}\right)\right)
\end{aligned}
$$

Here $A$ is defined as the set $\cup_{\left|G\left(B_{n}\right)\right| \leq n_{1}} B_{n}$.

$$
A=\cup_{\left|G\left(B_{n}\right)\right| \leq n_{1}} B_{n}
$$

So far, we proved the interaction potential between particles of the same groups cancels out the negative part of the $v_{2,1}^{a}\left(1-\chi_{A}\right)$ term in (2.79). We shall show that the kinetic energy and the positive part of $v_{2,1}^{a}$ cancel out the remaining negative part of $v_{2,1}^{a}$.

For the other terms in the Hamiltonian $H_{P}$, we claim that as long as

$$
S L\left[4 n_{1}\left(v+t v_{-}\right)\right] \geq 0
$$

we have

$$
\begin{aligned}
0 \leq & \frac{1}{2} \sum_{j \in \pi_{1}, i \in \pi_{2}}\left(1-F_{i, j}\right)\left(\left[v_{2,1}^{a}\right]_{+}\left(x_{i}-x_{j}\right)+\left[v_{2,1}^{a}\right]_{-}\left(x_{i}-x_{j}\right) \chi_{A}\left(x_{j}\right)\right) \\
& +\frac{2+t}{1+t} \sum_{j \in \pi_{1}}-\nabla_{j}\left(1-\sum_{i} F_{i, j}\right) \nabla_{j}
\end{aligned}
$$


As we can see that (2.95) and (2.98) implies that $H_{P} \geq 0$ when $S L\left[4 n_{1}\left(v+t v_{-}\right)\right] \geq 0$ and (2.92) holds, i.e., $\lambda_{+} \geq$const. $\left(1+t^{-1}\right) \lambda_{-}\left(R_{0} / r_{1}\right)^{3}$, which completes the proof of Theorem 2.

To prove (2.98), we only need to prove the following operator inequality, for any fixed $x_{2}, \cdots, x_{N}$,

$$
\begin{aligned}
0 \leq \quad & -\frac{2+t}{1+t} \nabla_{1}\left(1-\sum_{i=2}^{N} F_{i, 1}\right) \nabla_{1} \\
& +\frac{1}{2} \sum_{j \in \pi_{2}}\left(1-F_{j, 1}\right)\left(\left[v_{2,1}^{a}\right]_{+}\left(x_{1}-x_{j}\right)+\left[v_{2,1}^{a}\right]_{-}\left(x_{1}-x_{j}\right) \chi_{A}\left(x_{1}\right)\right)
\end{aligned}
$$

First, if $\left[v_{2,1}^{a}\right]_{-}\left(x_{1}-x_{j}\right) \chi_{A}\left(x_{1}\right) \neq 0$, then $d\left(B_{n}^{x_{1}}, x_{j}\right) \leq R_{0} a$, here the $B_{n}^{x_{1}}$ is the cube where $x_{1}$ is. We obtain that $j \in \pi_{2}^{\prime} \subset \pi_{2}$, here $\pi_{2}^{\prime}$ is defined as

$$
\pi_{2}^{\prime} \equiv\left\{j^{\prime} \in \pi_{2}: \exists B_{n}, D\left(x_{j^{\prime}}, B_{n}\right) \leq R_{0} a,\left|G\left(B_{n}\right)\right| \leq n_{1}\right\}
$$

Hence, it only remains to prove that

$$
0 \leq \quad-\frac{2+t}{1+t} \nabla_{1}\left(1-\sum_{i=2}^{N} F_{i, 1}\right) \nabla_{1}+\frac{1}{2} \sum_{j \in \pi_{2}^{\prime}}\left(1-F_{j, 1}\right) v_{2,1}^{a}\left(x_{1}-x_{j}\right)
$$

Second, we claim the following inequality which will be proved later.

$$
n_{1}\left(1-\sum_{i=2}^{N} F_{i, 1}\right) \geq \sum_{j \in \pi_{2}^{\prime}}\left(1-F_{j, 1}\right) \theta_{\left(R_{0} a\right)}\left(x_{1}-x_{j}\right)
$$

which implies that

$$
-\nabla_{1}\left(1-\sum_{i=2}^{N} F_{i, 1}\right) \nabla_{1} \geq-\frac{1}{n_{1}} \nabla_{1} \sum_{j \in \pi_{2}^{\prime}}\left(1-F_{j, 1}\right) \theta_{\left(R_{0} a\right)}\left(x_{1}-x_{j}\right) \nabla_{1}
$$

With (2.103), we obtain that the right side of (2.101) is not less than

$$
\begin{aligned}
& \sum_{j \in \pi_{2}^{\prime}}\left(-\frac{2+t}{n_{1}(1+t)} \nabla_{1}\left(1-F_{j, 1}\right) \theta_{\left(R_{0} a\right)}\left(x_{1}-x_{j}\right) \nabla_{1}+\frac{1}{2}\left(1-F_{j, 1}\right) v_{2,1}^{a}\left(x_{j}-x_{1}\right)\right) \\
\geq \quad & \sum_{j \in \pi_{2}^{\prime}}\left(1-\prod_{k \neq 1 \text { or } j} \bar{\theta}_{2 R}\left(x_{k}-x_{j}\right)\right) \times \frac{2}{n_{1}(1+t)} \\
& \times\left(-\nabla_{1} \theta_{\left(R_{0} a\right)}\left(x_{1}-x_{j}\right) \nabla_{1}+2 n_{1}\left(v^{a}+t v_{-}^{a}\right)\left(x_{j}-x_{1}\right)\right)
\end{aligned}
$$

Here we used the definition of $F_{j, 1}$ and (2.77), i.e., $v_{2,1}^{a}=\frac{4}{1+t}\left[v^{a}+t v_{-}^{a}\right]$. With the assumption $S L\left[4 n_{1}\left(v+v_{-}\right)\right] \geq 0$, we obtain that (2.104) $\geq 0$, which implies inequality (2.101). 
Hence, it only remains to prove (2.102). For $x_{2}, \cdots, x_{N}$ fixed, we define $\pi_{3}$ as following,

$$
\pi_{3}=\left\{2 \leq j \leq N: \prod_{2 \leq k \leq N, k \neq j} \bar{\theta}_{2 R}\left(x_{j}-x_{k}\right)=1\right\}
$$

With the definition of $\pi_{3}$, we obtain that

$$
F_{j, 1}= \begin{cases}\theta_{R}\left(x_{j}-x_{1}\right) & j \in \pi_{3} \\ 0 & j \notin \pi_{3}\end{cases}
$$

Hence, it only remains to prove that

$$
n_{1}\left(1-\sum_{i \in \pi_{3}} \theta_{R}\left(x_{1}-x_{i}\right)\right) \geq \sum_{j \in \pi_{2}^{\prime}, j \notin \pi_{3}} \theta_{\left(R_{0} a\right)}\left(x_{1}-x_{j}\right)
$$

or

$$
\max _{x \in \mathbb{R}^{3}}\left(n_{1} \sum_{i \in \pi_{3}} \theta_{R}\left(x-x_{i}\right)+\sum_{j \in \pi_{2}^{\prime}, j \notin \pi_{3}} \theta_{\left(R_{0} a\right)}\left(x-x_{j}\right)\right) \leq n_{1}
$$

Because the distance between $x_{i}\left(i \in \pi_{3}\right)$ and $x_{j}(2 \leq j \leq N, j \neq i)$ are not less than $2 R$, we have that if $i \in \pi_{3}$

$$
\theta_{R}\left(x-x_{i}\right)=1 \Rightarrow \sum_{j \neq 1, j \neq i} \theta_{R}\left(x-x_{j}\right)=0
$$

So, it only remains to prove that

$$
\max _{x}\left(\sum_{j \in \pi_{2}^{\prime}, j \notin \pi_{3}} \theta_{\left(R_{0} a\right)}\left(x-x_{j}\right)\right) \leq n_{1}
$$

By the definition of $\pi_{2}^{\prime}$ in (2.100), if $j \in \pi_{2}^{\prime}$ and $\theta_{\left(R_{0} a\right)}\left(x-x_{j}\right)=1$, there exist $B_{n}$ satisfying $\left|G\left(B_{n}\right)\right| \leq n_{1}$ and $d\left(x, B_{n}\right) \leq 2 R_{0} a$. Hence by the definition of $G\left(B_{n}\right)$ in (2.81) and (2.80), we obtain that, for $\forall x \in \mathbb{R}^{3}$

$$
\sum_{i \in \pi_{2}^{\prime}} \theta_{\left(R_{0} a\right)}\left(x-x_{i}\right)=1 \Rightarrow \sum_{i \in \pi_{2}} \theta_{\left(R_{0} a\right)}\left(x-x_{i}\right) \leq n_{1}
$$

With the fact that $\pi_{2}^{\prime} \subset \pi_{2}$, we arrive at the desired result (2.110) and complete the proof of Theorem 2 .

\subsection{Proof of Lemma 1}

Proof. Let $\delta \Omega$ be any infinitismal solid angle. With the definition of scattering length, we have that if $\phi$ is a complex-valued function such that

$$
\phi(x)=1 \text { for } x \in \delta \Omega \otimes \mathbb{R} \text { and }|x|=R^{\prime} \geq R_{0} a
$$


then

$$
\delta \Omega \cdot a \leq \int_{\delta \Omega \otimes\left[0, R^{\prime}\right]}|\nabla \phi(x)|^{2}+\frac{1}{2} v^{a}(x)|\phi(x)|^{2} d x
$$

Hence we obtain that

$$
a \int_{\mathbb{R}^{3}} \delta\left(|x|-R^{\prime}\right)|\phi(x)|^{2} d x \leq \int_{|x| \leq R^{\prime}}|\nabla \phi(x)|^{2}+\frac{1}{2} v^{a}(x)|\phi(x)|^{2} d x
$$

which says that for any non-negative radial function $U_{0}(x)$, supported in the annulus $R_{0} a \leq|x| \leq R$, with $\int_{\mathbb{R}^{3}} U_{0}(x) d x=4 \pi$, we have

$$
-\nabla \theta_{R}(x) \nabla+\frac{1}{2} v^{a} \geq a U_{0}
$$

Note: The result of lemma 2.5 of [5] shows the $\theta_{R}(x)$ in above inequality can be replaced with the characteristic function of any star-shaped set when $v^{a} \geq 0$.

Furthermore, one can easily prove that for fixed $v(S L[v]=1), v_{+}$and small enough $\varepsilon$,

$$
S L\left[v-\varepsilon v_{+}\right]>0, \quad\left|f_{\varepsilon}\right|_{\infty} \leq \text { const. }
$$

Here we denote $f_{\varepsilon}$ as the normalized solution $\left(\lim _{|x| \rightarrow \infty} f_{\varepsilon}(x)=1\right)$ of the zero-energy scattering equation of $v-\varepsilon v_{+}$. Hence, by the definition of scattering length, using $f_{\varepsilon}$ as the trial function for $v$, we obtain that

$$
S L[v] \leq S L\left[v-\varepsilon v_{+}\right]+\varepsilon\left\|v_{+}\right\|_{1} \cdot\left|f_{\varepsilon}\right|_{\infty} \leq S L\left[v-\varepsilon v_{+}\right]+\text {const. } \varepsilon
$$

Combining this result with (2.115) and the definition of $v_{\varepsilon}^{a}$, we have,

$$
-(1-\varepsilon) \nabla \theta_{R}(x) \nabla+\frac{1}{2} v_{\varepsilon}^{a} \geq(1-\text { const. } \varepsilon) a U_{0}
$$

and

$$
-(1-\varepsilon) \nabla_{j} F_{i, j} \nabla_{j}+\frac{1}{2} F_{i, j} v_{\varepsilon}^{a}\left(x_{i}-x_{j}\right) \geq(1-\text { const. } \varepsilon) a F_{i, j} U_{0}\left(x_{i}-x_{j}\right)
$$

Hence, we obtain the following lower bound on $H^{\prime \prime}$, which is defined in (2.76)

$$
H^{\prime \prime} \geq \varepsilon \sum_{j}-\Delta_{j}+\varepsilon \sum_{i \neq j} \frac{v_{+}^{a}}{2}\left(x_{i}-x_{j}\right)+(1-\text { const. } \varepsilon) a \sum_{i \neq j} W_{i, j}
$$

Here $W_{i, j}$ is defined as

$$
W_{i, j}=F_{i j} U_{0}\left(x_{i}-x_{j}\right) \geq 0
$$

As in [4, we choose

$$
\ell=a Y^{-6}
$$

and divide $\Lambda$ into small cubes with side length $\ell$. Then we have

$$
H^{\prime \prime} \geq H^{(3)} \equiv \varepsilon \sum_{j}-\Delta_{j}+\varepsilon \sum_{i \neq j} \frac{v_{+}^{a}}{2}\left(x_{i}-x_{j}\right)+(1-\text { const. } \varepsilon) a \sum_{i \neq j} W_{i, j}^{\prime}
$$


Here $W_{i, j}^{\prime}$ is defined as

$$
W_{i, j}^{\prime}=G_{i j} U_{0}\left(x_{i}-x_{j}\right), \quad G_{i j} \equiv F_{i j} \chi\left(x_{i}\right) \chi\left(x_{j}\right) \geq 0,
$$

and $\chi(x)$ is equal to 1 when the distance between $x$ and the edges of the small cubes is greater than $2 R$; otherwise it is equal to 0 . As we can see the particles in different cubes don't affect each other in $H^{(3)}$.

We are going to estimate the ground energy $E^{(3)}(n, \ell)$ of $H^{(3)}$ for $n$ particles in $[0, \ell]^{3}$ with Neumann boundary condition.

First, in the case that $n \leq \frac{8}{3} \varrho \ell^{3} Y^{-1}$, with the definition of $\varepsilon$ in (2.73), we have that

$$
H^{(3)} \quad \geq 3 Y \sum_{j}-\Delta_{j}+(1-\text { const. } Y) a \sum_{i \neq j} W_{i, j}^{\prime}
$$

Then with the Temple inequality in [10], as in [5] (Ineq. 2.60, 2.66), we have that

$$
\begin{aligned}
\frac{E^{(3)}(n, \ell)}{n} \geq & 4 \pi \frac{a n}{\ell^{3}}\left(1-\frac{1}{n}\right)(1-\text { const. } Y)\left(1-\frac{2 R}{\ell}\right)^{3}\left(1+\frac{4 \pi n}{3}\left(\frac{2 R}{\ell}\right)^{3}\right)^{-1} \\
& \left(1-\frac{3}{\pi} \frac{a n}{\left(R^{3}-\left(a R_{0}\right)^{3}\right)\left(3 \pi Y \ell^{-2}-4 a \ell^{-3} n^{2}\right)}\right) \\
\geq & 4 \pi \frac{a n}{\ell^{3}}\left(1-\frac{1}{n}\right)(1-\text { const. } Y)\left(1-\frac{n}{6 \ell^{3} \varrho} Y\right)
\end{aligned}
$$

Second, when $n \geq \frac{8}{3} \varrho \ell^{3} Y^{-1}$, using the fact $W^{\prime} \geq 0$, we obtain that

$$
H^{(3)} \geq \varepsilon H^{(4)} \equiv \varepsilon\left(\sum_{j}-\Delta_{j}+\sum_{i \neq j} \frac{v_{+}^{a}}{2}\left(x_{i}-x_{j}\right)\right)
$$

Using superadditivity of the ground state energy of $H^{(4)}$, we obtain that the ground energy $E^{(4)}(n, \ell)$ of $H^{(4)}$ is bounded from below as follows, $(n \geq p)$

$$
E^{(4)}(n, \ell) \geq\left[\frac{n}{p}\right] E^{(4)}(p, \ell) \geq \frac{n}{2 p} E^{(4)}(p, \ell)
$$

Here $[n / p]$ is the largest integer not greater than $n / p$. Actually, $H^{(4)}$ is just the Hamiltonian for the pure non-negative interaction potential, as in [7]. Denote $a_{+}$as follows:

$$
a_{+}=\min \left\{S L\left(v^{a}\right), S L\left(v_{+}^{a}\right)\right\} \leq a
$$

Replacing $v_{+}^{a}$ with soft potential, we obtain that,

$$
H^{(4)} \geq 3 Y \sum_{j}-\Delta_{j}+(1-\text { const. } Y) a_{+} \sum_{i \neq j} W_{i, j}^{\prime}
$$


As (2.126), we can prove that when $p=\frac{8}{3} \varrho \ell^{3} Y^{-1}$,

$$
E^{(4)}(p, \ell) / p \geq \frac{32}{3} \pi a_{+} \varrho Y^{-1}\left(1-\frac{1}{2}\right)(1-\text { const. } Y) \geq \frac{16}{3} \pi a_{+} \varrho Y^{-1}
$$

Hence when $n \geq \frac{8}{3} \varrho \ell^{3} Y^{-1}$, we have the following lower bound on the ground energy $E^{(3)}(n, \ell)$ of $H^{(3)}$

$$
E^{(3)}(n, \ell) / n \geq \varepsilon E^{(4)}(n, \ell) / n \geq \frac{8}{3} \pi \varepsilon a_{+} \varrho Y^{-1} \geq 8 \pi a \varrho
$$

For the last inequality, we used the definition of $\varepsilon$ in (2.73). So far we proved that

$$
\frac{E^{(3)}(n, \ell)}{n} \geq \begin{cases}4 \pi \frac{a n}{\ell^{3}}\left(1-\frac{1}{n}\right)(1-\text { const. } Y)\left(1-\frac{n}{6 \ell^{3} \varrho} Y\right) & 1 \leq n \leq \frac{8}{3} \ell^{3} \varrho Y^{-1} \\ 8 \pi a \varrho & n \geq \frac{8}{3} \ell^{3} \varrho Y^{-1}\end{cases}
$$

which implies that when $Y$ is small enough,

$$
\frac{E^{(3)}(n, \ell)}{n(1-\text { const. } Y)} \geq \begin{cases}4 \pi \frac{a n}{\ell^{3}}\left(1-\frac{1}{n}\right) & 1 \leq n \leq 4 \ell^{3} \varrho \\ 8 \pi a \varrho & n \geq 4 \ell^{3} \varrho\end{cases}
$$

Recall the following two facts,

1. the interaction potential $W_{i, j}^{\prime}$ only depends on the particles in the same cubes as $i$ and $j$,

2. the particles in different cubes have no interaction.

Using the inequality above (2.134), with the method in [7] (Ineq. 2.55, 2.56), one can prove that the ground state $E^{(3)}(N, \Lambda)$ of $H^{(3)}$ of $N$ particles in big cubic $\Lambda$ is greater than

$$
E^{(3)}(N, \Lambda) / N \geq 4 \pi a \varrho(1-\text { const. } Y) .
$$

Here $Y$ is defined in (2.72), which implies the desired result (2.76) .

\section{Appendix}

In this appendix, we show that if $v^{a}$ is a continuous function and $H_{N}$ has no bound state for any $N, v^{a}$ has a positive core and bounded from below, i.e.,

$$
v^{a}(0)>0, \quad \min v^{a}(r) \neq-\infty
$$

And these inequalities also hold when $v^{a}$ is stable [1] in the sense of (3.2). One can see that $\min v^{a}(r) \neq-\infty$ is trivial when $v^{a}$ is continuous. So it only remains to prove that $v^{a}(0)>0$.

First, we prove the statement in the case when $v^{a}$ is stable, which is defined as follows: there exists constant $C$, for any $N, x_{1}, \cdots, x_{N}$,

$$
\sum_{1 \leq i \neq j \leq N} v^{a}\left(x_{i}-x_{j}\right) \geq-C N
$$


Inserting

$$
x_{1}=x_{2}=\cdots=x_{[N / 2]}=0, \quad x_{[N / 2]+1}=x_{[N / 2]+2}=\cdots=x_{N}=x_{0}
$$

into the left side of (3.2), for some $x_{0} \in \mathbb{R}^{3}$ satisfying $v^{a}\left(x_{0}\right)<0$, we obtain that

$$
\text { const. } v^{a}(0) N^{2}-\text { const. } v^{a}\left(x_{0}\right) N^{2} \geq-C N,
$$

which implies the desired result that $v^{a}(0)>0$.

Next, we prove the statement in the case that $H_{N}$ has no bounded state for any $N$. Because $v^{a}$ is not pure non-negative, there exist $x_{0} \in \mathbb{R}^{3}, r_{1}, C \in R$ satisfying that

$$
v^{a}(x)<-C, \text { for } x \in B\left(x_{0}, r_{1}\right) \subset \mathbb{R}^{3}
$$

Here $B\left(x_{0}, r_{1}\right)$ is the sphere of radius $r_{0}$ centered at $x_{0}$. If $v^{a}(0) \leq 0$, there exists $r_{2}<r_{1} / 2$ satisfying that

$$
v^{a}(x)<C / 2, \text { for } x \in B\left(0, r_{2}\right)
$$

We construct the trial state such that $x_{1}, x_{2}, \cdots, x_{[N / 2]}$ are localized in $B\left(0, r_{2}\right)$ with the Dirichelet boundary condition and $x_{[N / 2]+1}, x_{[N / 2]+2}, \cdots, x_{N}$ are localized in $B\left(x_{0}, r_{2}\right)$ with the same boundary condition. The energy of this state is less than

$$
-\frac{C}{8} N^{2}+\frac{\text { const. }}{r_{2}^{2}} N
$$

Here the first term is potential energy and the second term is kinetic energy. When $N$ goes to infinity, the energy of this trial state is negative and hence there are bound states, which is a contradiction with our assumptions. So we arrive at the desired result that $v^{a}(0)>0$.

\section{References}

[1] D. Ruelle, Statistical Mechanics: Rigorous Results (1989).

[2] E.H. Lieb and R. Seiringer, Derivation of the Gross-Pitaevskii Equation for Rotating Bose Gases, Comm. Math. Phys. Volume 264, Issue 2, pp.505-537 (2005).

[3] E.H. Lieb, R. Seiringer, J.P. Solovej, Ground State Energy of the Low Density Fermi Gas, in: Recent Advances in Differential Equations and Mathematical Physics, N. Chernov, Y. Karpeshina, I. Knowles, R. Lewis, R. Weikard, eds., Amer. Math. Soc. Contemporary Math. series 412, 239-248 (2006)

[4] E.H. Lieb, R. Seiringer, J. P. Solovej, J. Yngvason The Ground State of the Bose Gas Current Developments in Mathematics, (2001), International Press, Cambridge, (2002), pp. 131-178. 
[5] E.H. Lieb, R. Seiringer, J. P. Solovej, J. Yngvason, The Mathematics of the Bose Gas and its Condensation, Oberwolfach Seminars Vol. 34, (2005).

[6] E.H. Lieb, R. Seiringer, J. Yngvason, Bosons in a Trap: A Rigorous Derivation of the Gross-Pitaevskii Energy Functional, Phys. Rev. A 61, 043602 (2000).

[7] E.H. Lieb, J. Yngvason, Ground State Energy of the Low Density Bose Gas, Phys. Rev. Lett. 80, 2504-2507 (1998).

[8] E.H. Lieb, J. Yngvason, The Ground State Energy of a Dilute Two-dimensional Bose Gas, J. Stat. Phys. 103, 509 (2001). arXiv math-ph/0002014, mp arc 0063.

[9] F.J. Dyson, Ground State Energy of a Hard-Sphere Gas, Phys. Rev. 106, 20-26 (1957).

[10] G. Temple, The theory of Rayleigh's Principle as Applied to Continuous Systems, Proc. Roy. Soc. London A 119, 276-293 (1928).

[11] L.J. Oon, Ground State Energy of Dilute Bose Gas in Small Negative Potential Case Arxiv: 0803.0533. 